Supplement of Atmos. Chem. Phys., 21, 6221-6230, 2021

https://doi.org/10.5194/acp-21-6221-2021-supplement

(C) Author(s) 2021. CC BY 4.0 License.

(c) (1)

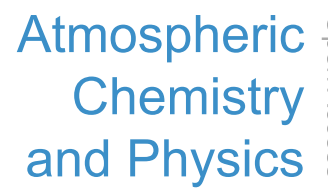

Atmospheric

and Physics

Supplement of

\title{
Influence of atmospheric conditions on the role of trifluoroacetic acid in atmospheric sulfuric acid-dimethylamine nucleation
}

Ling Liu et al.

Correspondence to: Xiuhui Zhang (zhangxiuhui@bit.edu.cn)

The copyright of individual parts of the supplement might differ from the article licence. 


\section{Section 1}

From the ratios of $\beta \cdot C / \Sigma \gamma$ for the studied SA-DMA-TFA clusters (Fig. 1), it can be seen that the $(\mathrm{SA})_{2} \cdot(\mathrm{DMA})_{3} \cdot(\mathrm{TFA})_{1}$ and $(\mathrm{SA})_{1} \cdot(\mathrm{DMA})_{2} \cdot(\mathrm{TFA})_{1}$ clusters are relatively stable against evaporation and can be able to grow into larger clusters. The (SA) $)_{1}$ (DMA) $)_{1}$ (TFA) $)_{1}$ cluster is the initial and key cluster to form $(\mathrm{SA})_{2} \cdot(\mathrm{DMA})_{3} \cdot(\mathrm{TFA})_{1}$ and $(\mathrm{SA})_{1} \cdot(\mathrm{DMA})_{2} \cdot(\mathrm{TFA})_{1}$ clusters as shown in Fig. 4, and this cluster formation pathway through $(\mathrm{SA})_{1} \cdot(\mathrm{DMA})_{1} \cdot(\mathrm{TFA})_{1}$ involves a modest thermodynamic barrier shown by the previous study ( $\mathrm{Lu}$ et al. 2020). Hence, the formation of $(\mathrm{SA})_{1} \cdot(\mathrm{DMA})_{1} \cdot(\mathrm{TFA})_{1}$ cluster is the limiting step in SA-DMA-TFA new particle formation, which is similar to the analysis of the limiting step of (SA) $)_{n}(\text { Base })_{n}$ system (Elm, 2017). Therefore, in order to understand the influence of relative humidity $(\mathrm{RH})$ on the SA-DMA-TFA system, the evaluation of the influence of $\mathrm{RH}$ on the formation kinetics of (SA) $)_{1} \cdot(\mathrm{DMA})_{1} \cdot(\mathrm{TFA})_{1}$ cluster is of significance. Herein, the kinetic property of hydrated clusters relevant to the formation of the hydrated $(\mathrm{SA})_{1} \cdot(\mathrm{DMA})_{1} \cdot(\mathrm{TFA})_{1}$ clusters, which involves collisions of smaller clusters with monomers and evaporation of monomers from larger clusters, has been studied. The relative hydrate distributions of the clusters (Tables S5-S6) and the effective collision coefficients and evaporation coefficients (Table S7) weighted average over the hydrate distributions (Paasonen et al., 2012) are calculated at $280 \mathrm{~K}$ and $260 \mathrm{~K}$. The Cartesian coordinates of these studied hydrated clusters are listed in Table S8. The relative distributions of the unhydrated (SA) $\cdot(\mathrm{DMA})_{1} \cdot(\mathrm{TFA})_{1}$ and $(\mathrm{SA})_{1} \cdot(\mathrm{DMA})_{2} \cdot(\mathrm{TFA})_{1}$ clusters are more than $50 \%$, which are higher than those of the corresponding hydrated clusters at different RHs (20\%, 40\%, 60\%, 80\%, and 100\%) and different temperatures $(280 \mathrm{~K}$ and $260 \mathrm{~K})$. Hence, most of $(\mathrm{SA})_{1} \cdot(\mathrm{DMA})_{1} \cdot(\mathrm{TFA})_{1}$ and $(\mathrm{SA})_{1} \cdot(\mathrm{DMA})_{2} \cdot(\mathrm{TFA})_{1}$ clusters are unhydrated in the atmosphere. Furthermore, the ratios of effective collision frequencies with nucleation monomers versus total effective evaporation frequencies $\left(\beta_{e f f} C \Sigma \gamma_{e f f}\right)$ of these two key clusters almost vary slightly within one order of magnitude at different RHs and different temperatures (Table S7). Therefore, the studied SADMA-TFA system is insensitive to the variation of humidity, which is similar to the SA-DMA system (Olenius et al., 2017). 


\section{Section 2}

Table S1. Gibbs free formation energies $(\Delta G, \mathrm{kcal} / \mathrm{mol})$ of the studied clusters at different temperatures at the RI-CC2/aug-cc-pV(T + d)Z//M06-2X/6-311++G(3df,3pd) level of theory.

\begin{tabular}{|c|c|c|}
\hline \multirow{2}{*}{ Clusters } & \multicolumn{2}{|c|}{$\Delta G(\mathrm{kcal} / \mathrm{mol})$} \\
\hline & $280 \mathrm{~K}$ (Lu et al., 2020) & $260 \mathrm{~K}$ \\
\hline$(\mathrm{TFA})_{2}$ & -5.76 & -6.51 \\
\hline$(\mathrm{TFA})_{3}$ & -3.79 & -5.24 \\
\hline$(\mathrm{SA})_{1} \cdot(\mathrm{TFA})_{1}$ & -7.83 & -8.53 \\
\hline$(\mathrm{SA})_{1} \cdot(\mathrm{TFA})_{2}$ & -13.49 & -15.03 \\
\hline$(\mathrm{SA})_{2} \cdot(\mathrm{TFA})_{1}$ & -14.68 & -16.27 \\
\hline$(\mathrm{DMA})_{1} \cdot(\mathrm{TFA})_{1}$ & -8.65 & -9.31 \\
\hline$(\mathrm{DMA})_{1} \cdot(\mathrm{TFA})_{2}$ & -19.06 & -20.57 \\
\hline$(\mathrm{DMA})_{1} \cdot(\mathrm{TFA})_{3}$ & -23.56 & -26.06 \\
\hline$(\mathrm{DMA})_{2} \cdot(\mathrm{TFA})_{2}$ & -34.55 & -36.88 \\
\hline$(\mathrm{SA})_{1} \cdot(\mathrm{DMA})_{1} \cdot(\mathrm{TFA})_{1}$ & -27.21 & -28.67 \\
\hline$(\mathrm{SA})_{2} \cdot(\mathrm{DMA})_{1} \cdot(\mathrm{TFA})_{1}$ & -42.13 & -44.48 \\
\hline$(\mathrm{SA})_{1} \cdot(\mathrm{DMA})_{2} \cdot(\mathrm{TFA})_{1}$ & -44.92 & -47.27 \\
\hline$(\mathrm{SA})_{1} \cdot(\mathrm{DMA})_{2} \cdot(\mathrm{TFA})_{2}$ & -52.41 & -55.74 \\
\hline$(\mathrm{SA})_{2} \cdot(\mathrm{DMA})_{2} \cdot(\mathrm{TFA})_{1}$ & -61.91 & -65.25 \\
\hline$(\mathrm{SA})_{1} \cdot(\mathrm{DMA})_{1} \cdot(\mathrm{TFA})_{2}$ & -36.32 & -38.57 \\
\hline$(\mathrm{SA})_{2}$ & -9.04 & -9.72 \\
\hline$(\mathrm{SA})_{3}$ & -15.33 & -16.87 \\
\hline$(\mathrm{SA})_{2} \cdot(\mathrm{DMA})_{1}$ & -36.30 & -37.85 \\
\hline$(\mathrm{SA})_{2} \cdot(\mathrm{DMA})_{2}$ & -56.95 & -59.26 \\
\hline$(\mathrm{SA})_{3} \cdot(\mathrm{DMA})_{1}$ & -50.06 & -52.44 \\
\hline$(\mathrm{SA})_{3} \cdot(\mathrm{DMA})_{2}$ & -73.77 & -76.90 \\
\hline$(\mathrm{SA})_{3} \cdot(\mathrm{DMA})_{3}$ & -94.24 & -98.18 \\
\hline$(\mathrm{SA})_{1} \cdot(\mathrm{DMA})_{1}$ & -15.28 & -15.98 \\
\hline$(\mathrm{SA})_{2} \cdot(\mathrm{DMA})_{3} \cdot(\mathrm{TFA})_{1}$ & -79.52 & -83.64 \\
\hline$(\mathrm{SA})_{1} \cdot(\mathrm{DMA})_{3} \cdot(\mathrm{TFA})_{2}$ & -68.39 & -72.51 \\
\hline$(\mathrm{DMA})_{3} \cdot(\mathrm{TFA})_{3}$ & -54.18 & -58.31 \\
\hline$(\mathrm{DMA})_{2} \cdot(\mathrm{TFA})_{3}$ & -43.13 & -46.42 \\
\hline
\end{tabular}


Table S2. Collision coefficients $\left(\beta, \mathrm{cm}^{3} \mathrm{~s}^{-1}\right)$ for each cluster in the present study.

\begin{tabular}{|c|c|c|}
\hline \multirow[b]{2}{*}{ Collisions } & \multicolumn{2}{|c|}{$\beta\left(\mathrm{cm}^{3} \mathrm{~s}^{-1}\right)$} \\
\hline & $\begin{array}{c}280 \mathrm{~K} \text { (Lu et al., } \\
2020)\end{array}$ & $260 \mathrm{~K}$ \\
\hline $\mathrm{DMA}+\mathrm{SA}$ & $2.45 \times 10^{-10}$ & $2.36 \times 10^{-10}$ \\
\hline$(\mathrm{SA})_{1} \cdot(\mathrm{DMA})_{1}+\mathrm{SA}$ & $2.67 \times 10^{-10}$ & $2.57 \times 10^{-10}$ \\
\hline$(\mathrm{SA})_{2} \cdot(\mathrm{DMA})_{1}+\mathrm{SA}$ & $2.61 \times 10^{-10}$ & $2.52 \times 10^{-10}$ \\
\hline$(\mathrm{SA})_{2} \cdot(\mathrm{DMA})_{1}+\mathrm{DMA}$ & $3.91 \times 10^{-10}$ & $3.77 \times 10^{-10}$ \\
\hline$(\mathrm{SA})_{2} \cdot(\mathrm{DMA})_{2}+\mathrm{SA}$ & $3.05 \times 10^{-10}$ & $2.94 \times 10^{-10}$ \\
\hline$(\mathrm{SA})_{3} \cdot(\mathrm{DMA})_{2}+\mathrm{DMA}$ & $5.82 \times 10^{-10}$ & $5.61 \times 10^{-10}$ \\
\hline $\mathrm{DMA}+\mathrm{TFA}$ & $2.85 \times 10^{-10}$ & $2.75 \times 10^{-10}$ \\
\hline$(\mathrm{SA})_{1} \cdot(\mathrm{DMA})_{1}+\mathrm{TFA}$ & $2.95 \times 10^{-10}$ & $2.84 \times 10^{-10}$ \\
\hline$(\mathrm{SA})_{2} \cdot(\mathrm{DMA})_{1}+\mathrm{TFA}$ & $2.84 \times 10^{-10}$ & $2.74 \times 10^{-10}$ \\
\hline $\mathrm{DMA}+(\mathrm{SA})_{1} \cdot(\mathrm{DMA})_{1} \cdot(\mathrm{TFA})_{1}$ & $4.39 \times 10^{-10}$ & $4.23 \times 10^{-10}$ \\
\hline$(\mathrm{SA})_{2} \cdot(\mathrm{DMA})_{2}+\mathrm{TFA}$ & $3.28 \times 10^{-10}$ & $3.16 \times 10^{-10}$ \\
\hline $\mathrm{DMA}+(\mathrm{SA})_{2} \cdot(\mathrm{DMA})_{2} \cdot(\mathrm{TFA})_{1}$ & $4.90 \times 10^{-10}$ & $4.72 \times 10^{-10}$ \\
\hline$(\mathrm{DMA})_{1} \cdot(\mathrm{TFA})_{1}+\mathrm{TFA}$ & $3.26 \times 10^{-10}$ & $3.15 \times 10^{-10}$ \\
\hline $\mathrm{TFA}+(\mathrm{SA})_{1} \cdot(\mathrm{DMA})_{1} \cdot(\mathrm{TFA})_{1}$ & $3.17 \times 10^{-10}$ & $3.05 \times 10^{-10}$ \\
\hline$(\mathrm{DMA})_{1} \cdot(\mathrm{TFA})_{2}+\mathrm{DMA}$ & $4.47 \times 10^{-10}$ & $4.31 \times 10^{-10}$ \\
\hline $\mathrm{TFA}+(\mathrm{SA})_{1} \cdot(\mathrm{DMA})_{2} \cdot(\mathrm{TFA})_{1}$ & $3.52 \times 10^{-10}$ & $3.39 \times 10^{-10}$ \\
\hline $\mathrm{DMA}+(\mathrm{SA})_{1} \cdot(\mathrm{DMA})_{2} \cdot(\mathrm{TFA})_{2}$ & $4.79 \times 10^{-10}$ & $4.62 \times 10^{-10}$ \\
\hline$(\mathrm{DMA})_{1} \cdot(\mathrm{TFA})_{2}+\mathrm{TFA}$ & $3.21 \times 10^{-10}$ & $3.10 \times 10^{-10}$ \\
\hline$(\mathrm{DMA})_{2} \cdot(\mathrm{TFA})_{2}+\mathrm{TFA}$ & $3.50 \times 10^{-10}$ & $3.38 \times 10^{-10}$ \\
\hline$(\mathrm{DMA})_{2} \cdot(\mathrm{TFA})_{3}+\mathrm{DMA}$ & $4.69 \times 10^{-10}$ & $4.52 \times 10^{-10}$ \\
\hline TFA+TFA & $2.25 \times 10^{-10}$ & $2.17 \times 10^{-10}$ \\
\hline$(\mathrm{TFA})_{2}+\mathrm{TFA}$ & $3.35 \times 10^{-10}$ & $3.23 \times 10^{-10}$ \\
\hline $\mathrm{SA}+\mathrm{TFA}$ & $1.97 \times 10^{-10}$ & $1.90 \times 10^{-10}$ \\
\hline$(\mathrm{SA})_{1} \cdot(\mathrm{TFA})_{1}+\mathrm{TFA}$ & $3.04 \times 10^{-10}$ & $2.93 \times 10^{-10}$ \\
\hline$(\mathrm{SA})_{2}+\mathrm{TFA}$ & $2.80 \times 10^{-10}$ & $2.69 \times 10^{-10}$ \\
\hline $\mathrm{SA}+\mathrm{SA}$ & $1.70 \times 10^{-10}$ & $1.64 \times 10^{-10}$ \\
\hline$(\mathrm{SA})_{2}+\mathrm{SA}$ & $2.55 \times 10^{-10}$ & $2.46 \times 10^{-10}$ \\
\hline$(\mathrm{TFA})_{2}+\mathrm{SA}$ & $3.11 \times 10^{-10}$ & $3.00 \times 10^{-10}$ \\
\hline$(\mathrm{TFA})_{3}+\mathrm{SA}$ & $3.38 \times 10^{-10}$ & $3.25 \times 10^{-10}$ \\
\hline$(\mathrm{SA})_{1} \cdot(\mathrm{TFA})_{1}+\mathrm{SA}$ & $2.79 \times 10^{-10}$ & $2.69 \times 10^{-10}$ \\
\hline$(\mathrm{SA})_{1} \cdot(\mathrm{TFA})_{2}+\mathrm{SA}$ & $4.54 \times 10^{-10}$ & $4.37 \times 10^{-10}$ \\
\hline$(\mathrm{SA})_{2} \cdot(\mathrm{TFA})_{1}+\mathrm{SA}$ & $3.86 \times 10^{-10}$ & $3.72 \times 10^{-10}$ \\
\hline$(\mathrm{DMA})_{1} \cdot(\mathrm{TFA})_{1}+\mathrm{SA}$ & $2.99 \times 10^{-10}$ & $2.88 \times 10^{-10}$ \\
\hline$(\mathrm{DMA})_{1} \cdot(\mathrm{TFA})_{2}+\mathrm{SA}$ & $2.99 \times 10^{-10}$ & $2.87 \times 10^{-10}$ \\
\hline$(\mathrm{DMA})_{1} \cdot(\mathrm{TFA})_{3}+\mathrm{SA}$ & $3.11 \times 10^{-10}$ & $3.00 \times 10^{-10}$ \\
\hline$(\mathrm{DMA})_{2} \cdot(\mathrm{TFA})_{2}+\mathrm{SA}$ & $3.28 \times 10^{-10}$ & $3.16 \times 10^{-10}$ \\
\hline$(\mathrm{DMA})_{3} \cdot(\mathrm{TFA})_{3}+\mathrm{SA}$ & $3.32 \times 10^{-10}$ & $3.20 \times 10^{-10}$ \\
\hline$(\mathrm{DMA})_{2} \cdot(\mathrm{TFA})_{3}+\mathrm{SA}$ & $3.06 \times 10^{-10}$ & $2.95 \times 10^{-10}$ \\
\hline$(\mathrm{SA})_{1} \cdot(\mathrm{DMA})_{1} \cdot(\mathrm{TFA})_{1}+\mathrm{SA}$ & $2.93 \times 10^{-10}$ & $2.83 \times 10^{-10}$ \\
\hline$(\mathrm{SA})_{2} \cdot(\mathrm{DMA})_{1} \cdot(\mathrm{TFA})_{1}+\mathrm{SA}$ & $3.95 \times 10^{-10}$ & $3.80 \times 10^{-10}$ \\
\hline$(\mathrm{SA})_{1} \cdot(\mathrm{DMA})_{2} \cdot(\mathrm{TFA})_{1}+\mathrm{SA}$ & $3.29 \times 10^{-10}$ & $3.17 \times 10^{-10}$ \\
\hline
\end{tabular}




\begin{tabular}{lll}
\hline$(\mathrm{SA})_{1} \cdot(\mathrm{DMA})_{2} \cdot(\mathrm{TFA})_{2}+\mathrm{SA}$ & $3.14 \times 10^{-10}$ & $3.02 \times 10^{-10}$ \\
$(\mathrm{SA})_{2} \cdot(\mathrm{DMA})_{2} \cdot(\mathrm{TFA})_{1}+\mathrm{SA}$ & $3.22 \times 10^{-10}$ & $3.10 \times 10^{-10}$ \\
$(\mathrm{SA})_{1} \cdot(\mathrm{DMA})_{1} \cdot(\mathrm{TFA})_{2}+\mathrm{SA}$ & $4.31 \times 10^{-10}$ & $4.15 \times 10^{-10}$ \\
$(\mathrm{SA})_{2} \cdot(\mathrm{DMA})_{3} \cdot(\mathrm{TFA})_{1}+\mathrm{SA}$ & $3.41 \times 10^{-10}$ & $3.29 \times 10^{-10}$ \\
$(\mathrm{SA})_{1} \cdot(\mathrm{DMA})_{3} \cdot(\mathrm{TFA})_{2}+\mathrm{SA}$ & $3.41 \times 10^{-10}$ & $3.28 \times 10^{-10}$ \\
$(\mathrm{TFA})_{2}+\mathrm{DMA}$ & $4.60 \times 10^{-10}$ & $4.43 \times 10^{-10}$ \\
$(\mathrm{TFA})_{3}+\mathrm{DMA}$ & $5.10 \times 10^{-10}$ & $4.91 \times 10^{-10}$ \\
$(\mathrm{SA})_{1} \cdot(\mathrm{TFA})_{1}+\mathrm{DMA}$ & $4.14 \times 10^{-10}$ & $3.99 \times 10^{-10}$ \\
$(\mathrm{SA})_{1} \cdot(\mathrm{TFA})_{2}+\mathrm{DMA}$ & $6.75 \times 10^{-10}$ & $6.51 \times 10^{-10}$ \\
$(\mathrm{SA})_{2} \cdot(\mathrm{TFA})_{1}+\mathrm{DMA}$ & $5.76 \times 10^{-10}$ & $5.55 \times 10^{-10}$ \\
$(\mathrm{DMA})_{1} \cdot(\mathrm{TFA})_{3}+\mathrm{DMA}$ & $4.74 \times 10^{-10}$ & $4.56 \times 10^{-10}$ \\
$(\mathrm{SA})_{2} \cdot(\mathrm{DMA})_{1} \cdot(\mathrm{TFA})_{1}+\mathrm{DMA}$ & $5.93 \times 10^{-10}$ & $5.71 \times 10^{-10}$ \\
$(\mathrm{SA})_{1} \cdot(\mathrm{DMA})_{1} \cdot(\mathrm{TFA})_{2}+\mathrm{DMA}$ & $6.46 \times 10^{-10}$ & $6.23 \times 10^{-10}$ \\
$(\mathrm{SA})_{1} \cdot(\mathrm{DMA})_{1} \cdot(\mathrm{TFA})_{1}+(\mathrm{SA})_{1} \cdot(\mathrm{DMA})_{1}$ & $3.80 \times 10^{-10}$ & $3.66 \times 10^{-10}$ \\
$(\mathrm{SA})_{1} \cdot(\mathrm{DMA})_{2} \cdot(\mathrm{TFA})_{1}+(\mathrm{SA})_{1} \cdot(\mathrm{DMA})_{1}$ & $4.14 \times 10^{-10}$ & $3.99 \times 10^{-10}$ \\
\hline
\end{tabular}


Table S3. Total evaporation coefficients $\left(\Sigma \gamma, \mathrm{s}^{-1}\right)$ for each cluster in the present study.

\begin{tabular}{lcc}
\hline \multirow{2}{*}{ Clusters } & \multicolumn{2}{c}{$\Sigma \gamma\left(\mathrm{s}^{-1}\right)$} \\
\cline { 2 - 3 } & $280 \mathrm{~K}(\mathrm{Lu} \mathrm{et} \mathrm{al.,} 2020)$ & $260 \mathrm{~K}$ \\
\hline$(\mathrm{TFA})_{2}$ & $9.40 \times 10^{4}$ & $1.03 \times 10^{4}$ \\
$(\mathrm{TFA})_{3}$ & $3.03 \times 10^{11}$ & $1.06 \times 10^{11}$ \\
$(\mathrm{SA})_{1} \cdot(\mathrm{TFA})_{1}$ & $4.00 \times 10^{3}$ & $3.63 \times 10^{2}$ \\
$(\mathrm{SA})_{1} \cdot(\mathrm{TFA})_{2}$ & $7.54 \times 10^{3}$ & $5.82 \times 10^{2}$ \\
$(\mathrm{SA})_{2} \cdot(\mathrm{TFA})_{1}$ & $2.90 \times 10^{5}$ & $2.37 \times 10^{4}$ \\
$(\mathrm{DMA})_{1} \cdot(\mathrm{TFA})_{1}$ & $1.32 \times 10^{3}$ & $1.16 \times 10^{2}$ \\
$(\mathrm{DMA})_{1} \cdot(\mathrm{TFA})_{2}$ & $6.41 \times 10^{1}$ & 3.05 \\
$(\mathrm{DMA})_{1} \cdot(\mathrm{TFA})_{3}$ & $2.59 \times 10^{6}$ & $2.12 \times 10^{5}$ \\
$(\mathrm{DMA})_{2} \cdot(\mathrm{TFA})_{2}$ & $9.53 \times 10^{-3}$ & $2.38 \times 10^{-4}$ \\
$(\mathrm{SA})_{1} \cdot(\mathrm{DMA})_{1} \cdot(\mathrm{TFA})_{1}$ & 3.77 & $1.73 \times 10^{-1}$ \\
$(\mathrm{SA})_{2} \cdot(\mathrm{DMA})_{1} \cdot(\mathrm{TFA})_{1}$ & $2.10 \times 10^{5}$ & $2.07 \times 10^{4}$ \\
$(\mathrm{SA})_{1} \cdot(\mathrm{DMA})_{2} \cdot(\mathrm{TFA})_{1}$ & $1.73 \times 10^{-4}$ & $2.77 \times 10^{-6}$ \\
$(\mathrm{SA})_{1} \cdot(\mathrm{DMA})_{2} \cdot(\mathrm{TFA})_{2}$ & $1.31 \times 10^{4}$ & $7.27 \times 10^{2}$ \\
$(\mathrm{SA})_{2} \cdot(\mathrm{DMA})_{2} \cdot(\mathrm{TFA})_{1}$ & $1.16 \times 10^{6}$ & $8.23 \times 10^{4}$ \\
$(\mathrm{SA})_{1} \cdot(\mathrm{DMA})_{1} \cdot(\mathrm{TFA})_{2}$ & $6.44 \times 10^{2}$ & $4.11 \times 10^{1}$ \\
$(\mathrm{SA})_{2}$ & $1.96 \times 10^{2}$ & $1.56 \times 10^{1}$ \\
$(\mathrm{SA})_{3}$ & $8.23 \times 10^{4}$ & $6.77 \times 10^{3}$ \\
$(\mathrm{SA})_{2} \cdot(\mathrm{DMA})_{1}$ & $2.75 \times 10^{-7}$ & $3.01 \times 10^{-9}$ \\
$(\mathrm{SA})_{2} \cdot(\mathrm{DMA})_{2}$ & $7.82 \times 10^{-7}$ & $1.07 \times 10^{-8}$ \\
$(\mathrm{SA})_{3} \cdot(\mathrm{DMA})_{1}$ & $1.25 \times 10^{-1}$ & $3.87 \times 10^{-3}$ \\
$(\mathrm{SA})_{3} \cdot(\mathrm{DMA})_{2}$ & $5.95 \times 10^{-4}$ & $1.24 \times 10^{-5}$ \\
$(\mathrm{SA})_{3} \cdot(\mathrm{DMA})_{3}$ & $1.61 \times 10^{-6}$ & $2.05 \times 10^{-8}$ \\
$(\mathrm{SA})_{1} \cdot(\mathrm{DMA})_{1}$ & $7.62 \times 10^{-3}$ & $2.47 \times 10^{-4}$ \\
$(\mathrm{SA})_{2} \cdot(\mathrm{DMA})_{3} \cdot(\mathrm{TFA})_{1}$ & $2.31 \times 10^{-4}$ & $4.64 \times 10^{-6}$ \\
$(\mathrm{SA})_{1} \cdot(\mathrm{DMA})_{3} \cdot(\mathrm{TFA})_{2}$ & $4.23 \times 10^{-3}$ & $1.04 \times 10^{-4}$ \\
$(\mathrm{DMA})_{3} \cdot(\mathrm{TFA})_{3}$ & $2.92 \times 10^{1}$ & \\
$(\mathrm{DMA})_{2} \cdot(\mathrm{TFA})_{3}$ & $1.85 \times 10^{3}$ & \\
\hline & & \\
\hline
\end{tabular}


Table S4. Ratios $(\beta C / \Sigma \gamma)$ between monomer molecule collisions and evaporation coefficients for each cluster involving TFA in the present study. $[\mathrm{SA}]=1.0 \times 10^{6}$ molecules $\mathrm{cm}^{-3},[\mathrm{DMA}]=1.0 \times 10^{7}$ molecules $\mathrm{cm}^{-3}$, [TFA $]=1.0 \times 10^{6}$ molecules $\mathrm{cm}^{-3}$.

\begin{tabular}{|c|c|c|}
\hline \multirow{2}{*}{ Clusters } & \multicolumn{2}{|c|}{$\beta C / \Sigma \gamma$} \\
\hline & $280 \mathrm{~K}$ & $260 \mathrm{~K}$ \\
\hline \multicolumn{3}{|c|}{ Collision with SA monomer: $C=[\mathrm{SA}]$} \\
\hline $\begin{array}{l}(\mathrm{TFA})_{2} \\
(\mathrm{TFA})_{3} \\
(\mathrm{SA})_{1} \cdot(\mathrm{TFA})_{1} \\
(\mathrm{SA})_{1} \cdot(\mathrm{TFA})_{2} \\
(\mathrm{SA})_{2} \cdot(\mathrm{TFA})_{1} \\
(\mathrm{DMA})_{1} \cdot(\mathrm{TFA})_{1} \\
(\mathrm{DMA})_{1} \cdot(\mathrm{TFA})_{2} \\
(\mathrm{DMA})_{1} \cdot(\mathrm{TFA})_{3} \\
(\mathrm{DMA})_{2} \cdot(\mathrm{TFA})_{2} \\
(\mathrm{DMA})_{3} \cdot(\mathrm{TFA})_{3} \\
(\mathrm{DMA})_{2} \cdot(\mathrm{TFA})_{3} \\
(\mathrm{SA})_{1} \cdot(\mathrm{DMA})_{1} \cdot(\mathrm{TFA})_{1} \\
(\mathrm{SA})_{2} \cdot(\mathrm{DMA})_{1} \cdot(\mathrm{TFA})_{1} \\
(\mathrm{SA})_{1} \cdot(\mathrm{DMA})_{2} \cdot(\mathrm{TFA})_{1} \\
(\mathrm{SA})_{1} \cdot(\mathrm{DMA})_{2} \cdot(\mathrm{TFA})_{2} \\
(\mathrm{SA})_{2} \cdot(\mathrm{DMA})_{2} \cdot(\mathrm{TFA})_{1} \\
(\mathrm{SA})_{1} \cdot(\mathrm{DMA})_{1} \cdot(\mathrm{TFA})_{2} \\
(\mathrm{SA})_{2} \cdot(\mathrm{DMA})_{3} \cdot(\mathrm{TFA})_{1} \\
(\mathrm{SA})_{1} \cdot(\mathrm{DMA})_{3} \cdot(\mathrm{TFA})_{2}\end{array}$ & $\begin{array}{l}3.30 \times 10^{-9} \\
1.11 \times 10^{-15} \\
6.98 \times 10^{-8} \\
1.49 \times 10^{-9} \\
1.33 \times 10^{-9} \\
2.26 \times 10^{-7} \\
4.65 \times 10^{-6} \\
1.20 \times 10^{-10} \\
3.45 \times 10^{-2} \\
1.14 \times 10^{-5} \\
1.66 \times 10^{-7} \\
7.77 \times 10^{-5} \\
1.88 \times 10^{-9} \\
1.91 \\
2.39 \times 10^{-8} \\
2.78 \times 10^{-10} \\
6.69 \times 10^{-7} \\
1.48 \\
8.05 \times 10^{-2}\end{array}$ & $\begin{array}{c}2.90 \times 10^{-8} \\
3.06 \times 10^{-15} \\
7.41 \times 10^{-7} \\
1.54 \times 10^{-8} \\
1.57 \times 10^{-8} \\
2.48 \times 10^{-6} \\
9.44 \times 10^{-5} \\
1.41 \times 10^{-9} \\
1.33 \\
2.48 \times 10^{-4} \\
3.23 \times 10^{-6} \\
1.64 \times 10^{-3} \\
1.84 \times 10^{-8} \\
1.15 \times 10^{2} \\
4.16 \times 10^{-7} \\
3.77 \times 10^{-9} \\
1.01 \times 10^{-5} \\
7.08 \times 10^{1} \\
3.14\end{array}$ \\
\hline \multicolumn{3}{|c|}{ Collision with DMA monomer: $C=[\mathrm{DMA}]$} \\
\hline $\begin{array}{l}(\mathrm{TFA})_{2} \\
(\mathrm{TFA})_{3} \\
(\mathrm{SA})_{1} \cdot(\mathrm{TFA})_{1} \\
(\mathrm{SA})_{1} \cdot(\mathrm{TFA})_{2} \\
(\mathrm{SA})_{2} \cdot(\mathrm{TFA})_{1} \\
(\mathrm{DMA})_{1} \cdot(\mathrm{TFA})_{2} \\
(\mathrm{DMA})_{1} \cdot(\mathrm{TFA})_{3} \\
(\mathrm{DMA})_{2} \cdot(\mathrm{TFA})_{3} \\
(\mathrm{SA})_{1} \cdot(\mathrm{DMA})_{1} \cdot(\mathrm{TFA})_{1} \\
(\mathrm{SA})_{2} \cdot(\mathrm{DMA})_{1} \cdot(\mathrm{TFA})_{1} \\
(\mathrm{SA})_{1} \cdot(\mathrm{DMA})_{2} \cdot(\mathrm{TFA})_{2} \\
(\mathrm{SA})_{2} \cdot(\mathrm{DMA})_{2} \cdot(\mathrm{TFA})_{1} \\
(\mathrm{SA})_{1} \cdot(\mathrm{DMA})_{1} \cdot(\mathrm{TFA})_{2}\end{array}$ & $\begin{array}{l}4.89 \times 10^{-8} \\
1.68 \times 10^{-14} \\
1.03 \times 10^{-6} \\
2.22 \times 10^{-8} \\
1.98 \times 10^{-8} \\
6.98 \times 10^{-5} \\
1.83 \times 10^{-9} \\
2.54 \times 10^{-6} \\
1.16 \times 10^{-3} \\
2.82 \times 10^{-8} \\
3.65 \times 10^{-7} \\
4.24 \times 10^{-9} \\
1.00 \times 10^{-5}\end{array}$ & $\begin{array}{l}4.30 \times 10^{-7} \\
4.61 \times 10^{-14} \\
1.10 \times 10^{-5} \\
2.29 \times 10^{-7} \\
2.34 \times 10^{-7} \\
1.42 \times 10^{-3} \\
2.15 \times 10^{-8} \\
4.95 \times 10^{-5} \\
2.45 \times 10^{-2} \\
2.76 \times 10^{-7} \\
6.35 \times 10^{-6} \\
5.74 \times 10^{-8} \\
1.52 \times 10^{-4}\end{array}$ \\
\hline $\begin{array}{l}\text { Collision with TFA mor } \\
(\text { TFA })_{2} \\
(\mathrm{SA})_{1} \cdot(\mathrm{TFA})_{1} \\
(\mathrm{DMA})_{1} \cdot(\mathrm{TFA})_{1}\end{array}$ & $\begin{array}{l}3.56 \times 10^{-9} \\
7.59 \times 10^{-8} \\
2.46 \times 10^{-7}\end{array}$ & $\begin{array}{l}3.13 \times 10^{-8} \\
8.07 \times 10^{-7} \\
2.71 \times 10^{-6}\end{array}$ \\
\hline
\end{tabular}




\begin{tabular}{lcc}
\hline$(\text { DMA })_{1} \cdot(\text { TFA })_{2}$ & $5.01 \times 10^{-6}$ & $1.02 \times 10^{-4}$ \\
$(\text { DMA })_{2} \cdot(\text { TFA })_{2}$ & $3.68 \times 10^{-2}$ & 1.42 \\
$(\mathrm{SA})_{1} \cdot(\mathrm{DMA})_{1} \cdot(\mathrm{TFA})_{1}$ & $8.39 \times 10^{-5}$ & $1.77 \times 10^{-3}$ \\
\hline
\end{tabular}


Table S5. Relative hydrate distributions of the clusters at varying relative humidities (RHs) at 280 K.

\begin{tabular}{|c|c|c|c|c|c|}
\hline \multirow[t]{2}{*}{ Clusters } & \multicolumn{5}{|c|}{ Relative hydrate distributions } \\
\hline & $\mathrm{RH}=20 \%$ & $\mathrm{RH}=40 \%$ & $\mathrm{RH}=60 \%$ & $\mathrm{RH}=80 \%$ & $\mathrm{RH}=100 \%$ \\
\hline SA & $100 \%$ & $99 \%$ & $97 \%$ & $94 \%$ & $89 \%$ \\
\hline$(\mathrm{SA})_{1} \cdot\left(\mathrm{H}_{2} \mathrm{O}\right)_{1}$ & $0 \%$ & $0 \%$ & $0 \%$ & $0 \%$ & $0 \%$ \\
\hline$(\mathrm{SA})_{1} \cdot\left(\mathrm{H}_{2} \mathrm{O}\right)_{2}$ & $0 \%$ & $0 \%$ & $0 \%$ & $0 \%$ & $0 \%$ \\
\hline$(\mathrm{SA})_{1} \cdot\left(\mathrm{H}_{2} \mathrm{O}\right)_{3}$ & $0 \%$ & $1 \%$ & $3 \%$ & $6 \%$ & $11 \%$ \\
\hline TFA & $90 \%$ & $80 \%$ & $72 \%$ & $65 \%$ & $59 \%$ \\
\hline$(\mathrm{TFA})_{1} \cdot\left(\mathrm{H}_{2} \mathrm{O}\right)_{1}$ & $10 \%$ & $18 \%$ & $24 \%$ & $29 \%$ & $33 \%$ \\
\hline$(\mathrm{TFA})_{1} \cdot\left(\mathrm{H}_{2} \mathrm{O}\right)_{2}$ & $0 \%$ & $2 \%$ & $4 \%$ & $6 \%$ & $8 \%$ \\
\hline$(\mathrm{TFA})_{1} \cdot\left(\mathrm{H}_{2} \mathrm{O}\right)_{3}$ & $0 \%$ & $0 \%$ & $0 \%$ & $0 \%$ & $0 \%$ \\
\hline$(\mathrm{SA})_{1} \cdot(\mathrm{TFA})_{1}$ & $72 \%$ & $52 \%$ & $39 \%$ & $30 \%$ & $23 \%$ \\
\hline$(\mathrm{SA})_{1} \cdot(\mathrm{TFA})_{1} \cdot\left(\mathrm{H}_{2} \mathrm{O}\right)_{1}$ & $23 \%$ & $34 \%$ & $39 \%$ & $39 \%$ & $38 \%$ \\
\hline$(\mathrm{SA})_{1} \cdot(\mathrm{TFA})_{1} \cdot\left(\mathrm{H}_{2} \mathrm{O}\right)_{2}$ & $5 \%$ & $14 \%$ & $22 \%$ & $30 \%$ & $37 \%$ \\
\hline$(\mathrm{SA})_{1} \cdot(\mathrm{TFA})_{1} \cdot\left(\mathrm{H}_{2} \mathrm{O}\right)_{3}$ & $0 \%$ & $0 \%$ & $0 \%$ & $1 \%$ & $2 \%$ \\
\hline$(\mathrm{DMA})_{1} \cdot(\mathrm{TFA})_{1}$ & $12 \%$ & $6 \%$ & $4 \%$ & $3 \%$ & $2 \%$ \\
\hline$(\mathrm{DMA})_{1} \cdot(\mathrm{TFA})_{1} \cdot\left(\mathrm{H}_{2} \mathrm{O}\right)_{1}$ & $87 \%$ & $92 \%$ & $92 \%$ & $92 \%$ & $92 \%$ \\
\hline$(\mathrm{DMA})_{1} \cdot(\mathrm{TFA})_{1} \cdot\left(\mathrm{H}_{2} \mathrm{O}\right)_{2}$ & $1 \%$ & $2 \%$ & $4 \%$ & $5 \%$ & $6 \%$ \\
\hline$(\mathrm{SA})_{1} \cdot(\mathrm{DMA})_{1} \cdot(\mathrm{TFA})_{1}$ & $87 \%$ & $76 \%$ & $68 \%$ & $61 \%$ & $56 \%$ \\
\hline$(\mathrm{SA})_{1} \cdot(\mathrm{DMA})_{1} \cdot(\mathrm{TFA})_{1} \cdot\left(\mathrm{H}_{2} \mathrm{O}\right)_{1}$ & $13 \%$ & $23 \%$ & $31 \%$ & $37 \%$ & $42 \%$ \\
\hline$(\mathrm{SA})_{1} \cdot(\mathrm{DMA})_{1} \cdot(\mathrm{TFA})_{1} \cdot\left(\mathrm{H}_{2} \mathrm{O}\right)_{2}$ & $0 \%$ & $1 \%$ & $1 \%$ & $2 \%$ & $2 \%$ \\
\hline$(\mathrm{SA})_{1} \cdot(\mathrm{DMA})_{2} \cdot(\mathrm{TFA})_{1}$ & $95 \%$ & $90 \%$ & $85 \%$ & $81 \%$ & $77 \%$ \\
\hline$(\mathrm{SA})_{1} \cdot(\mathrm{DMA})_{2} \cdot(\mathrm{TFA})_{1} \cdot\left(\mathrm{H}_{2} \mathrm{O}\right)_{1}$ & $5 \%$ & $10 \%$ & $14 \%$ & $17 \%$ & $20 \%$ \\
\hline$(\mathrm{SA})_{1} \cdot(\mathrm{DMA})_{2} \cdot(\mathrm{TFA})_{1} \cdot\left(\mathrm{H}_{2} \mathrm{O}\right)_{2}$ & $0 \%$ & $0 \%$ & $1 \%$ & $2 \%$ & $3 \%$ \\
\hline
\end{tabular}


Table S6. Relative hydrate distributions of the clusters at varying relative humidities (RHs) at 260 $\mathrm{K}$.

\begin{tabular}{|c|c|c|c|c|c|}
\hline \multirow[t]{2}{*}{ Clusters } & \multicolumn{5}{|c|}{ Relative hydrate distributions } \\
\hline & $\mathrm{RH}=20 \%$ & $\mathrm{RH}=40 \%$ & $\mathrm{RH}=60 \%$ & $\mathrm{RH}=80 \%$ & $\mathrm{RH}=100 \%$ \\
\hline SA & $100 \%$ & $99 \%$ & $96 \%$ & $92 \%$ & $85 \%$ \\
\hline$(\mathrm{SA})_{1} \cdot\left(\mathrm{H}_{2} \mathrm{O}\right)_{1}$ & $0 \%$ & $0 \%$ & $0 \%$ & $0 \%$ & $0 \%$ \\
\hline$(\mathrm{SA})_{1} \cdot\left(\mathrm{H}_{2} \mathrm{O}\right)_{2}$ & $0 \%$ & $0 \%$ & $0 \%$ & $0 \%$ & $0 \%$ \\
\hline$(\mathrm{SA})_{1} \cdot\left(\mathrm{H}_{2} \mathrm{O}\right)_{3}$ & $0 \%$ & $1 \%$ & $4 \%$ & $8 \%$ & $15 \%$ \\
\hline TFA & $90 \%$ & $80 \%$ & $72 \%$ & $65 \%$ & $59 \%$ \\
\hline$(\mathrm{TFA})_{1} \cdot\left(\mathrm{H}_{2} \mathrm{O}\right)_{1}$ & $10 \%$ & $18 \%$ & $24 \%$ & $29 \%$ & $33 \%$ \\
\hline$(\mathrm{TFA})_{1} \cdot\left(\mathrm{H}_{2} \mathrm{O}\right)_{2}$ & $0 \%$ & $2 \%$ & $4 \%$ & $6 \%$ & $8 \%$ \\
\hline$(\mathrm{TFA})_{1} \cdot\left(\mathrm{H}_{2} \mathrm{O}\right)_{3}$ & $0 \%$ & $0 \%$ & $0 \%$ & $0 \%$ & $0 \%$ \\
\hline$(\mathrm{SA})_{1} \cdot(\mathrm{TFA})_{1}$ & $68 \%$ & $47 \%$ & $34 \%$ & $25 \%$ & $19 \%$ \\
\hline$(\mathrm{SA})_{1} \cdot(\mathrm{TFA})_{1} \cdot\left(\mathrm{H}_{2} \mathrm{O}\right)_{1}$ & $26 \%$ & $36 \%$ & $39 \%$ & $39 \%$ & $37 \%$ \\
\hline$(\mathrm{SA})_{1} \cdot(\mathrm{TFA})_{1} \cdot\left(\mathrm{H}_{2} \mathrm{O}\right)_{2}$ & $6 \%$ & $17 \%$ & $27 \%$ & $35 \%$ & $42 \%$ \\
\hline$(\mathrm{SA})_{1} \cdot(\mathrm{TFA})_{1} \cdot\left(\mathrm{H}_{2} \mathrm{O}\right)_{3}$ & $0 \%$ & $0 \%$ & $0 \%$ & $1 \%$ & $1 \%$ \\
\hline$(\mathrm{DMA})_{1} \cdot(\mathrm{TFA})_{1}$ & $8 \%$ & $4 \%$ & $3 \%$ & $2 \%$ & $1 \%$ \\
\hline$(\text { DMA })_{1} \cdot(\text { TFA })_{1} \cdot\left(\mathrm{H}_{2} \mathrm{O}\right)_{1}$ & $91 \%$ & $94 \%$ & $94 \%$ & $94 \%$ & $94 \%$ \\
\hline$(\text { DMA })_{1} \cdot(\mathrm{TFA})_{1} \cdot\left(\mathrm{H}_{2} \mathrm{O}\right)_{2}$ & $1 \%$ & $2 \%$ & $3 \%$ & $4 \%$ & $5 \%$ \\
\hline$(\mathrm{SA})_{1} \cdot(\mathrm{DMA})_{1} \cdot(\mathrm{TFA})_{1}$ & $85 \%$ & $74 \%$ & $65 \%$ & $58 \%$ & $53 \%$ \\
\hline$(\mathrm{SA})_{1} \cdot(\mathrm{DMA})_{1} \cdot(\mathrm{TFA})_{1} \cdot\left(\mathrm{H}_{2} \mathrm{O}\right)_{1}$ & $15 \%$ & $25 \%$ & $34 \%$ & $40 \%$ & $45 \%$ \\
\hline$(\mathrm{SA})_{1} \cdot(\mathrm{DMA})_{1} \cdot(\mathrm{TFA})_{1} \cdot\left(\mathrm{H}_{2} \mathrm{O}\right)_{2}$ & $0 \%$ & $1 \%$ & $1 \%$ & $2 \%$ & $2 \%$ \\
\hline$(\mathrm{SA})_{1} \cdot(\mathrm{DMA})_{2} \cdot(\mathrm{TFA})_{1}$ & $95 \%$ & $90 \%$ & $85 \%$ & $80 \%$ & $76 \%$ \\
\hline$(\mathrm{SA})_{1} \cdot(\mathrm{DMA})_{2} \cdot(\mathrm{TFA})_{1} \cdot\left(\mathrm{H}_{2} \mathrm{O}\right)_{1}$ & $5 \%$ & $10 \%$ & $14 \%$ & $18 \%$ & $21 \%$ \\
\hline$(\mathrm{SA})_{1} \cdot(\mathrm{DMA})_{2} \cdot(\mathrm{TFA})_{1} \cdot\left(\mathrm{H}_{2} \mathrm{O}\right)_{2}$ & $0 \%$ & $0 \%$ & $1 \%$ & $2 \%$ & $3 \%$ \\
\hline
\end{tabular}


Table S7. Ratios $(\beta C / \Sigma \gamma)$ between monomer molecule collisions and evaporation coefficients for the key clusters involving TFA in the present study at different relative humidities (RHs) and different temperatures. $C=1.0 \times 10^{8}$ molecules $\mathrm{cm}^{-3}$.

\begin{tabular}{|c|c|c|c|c|c|c|}
\hline \multirow{3}{*}{ Clusters } & \multicolumn{6}{|c|}{$\beta C / \Sigma \gamma$} \\
\hline & $\mathrm{RH}=0 \%$ & $\mathrm{RH}=20 \%$ & $\mathrm{RH}=40 \%$ & $\mathrm{RH}=60 \%$ & $\mathrm{RH}=80 \%$ & $\mathrm{RH}=100 \%$ \\
\hline & \multicolumn{6}{|c|}{$280 \mathrm{~K}$} \\
\hline $\begin{array}{l}(\mathrm{SA})_{1} \cdot(\mathrm{TFA})_{1} \\
(\mathrm{DMA})_{1} \cdot(\mathrm{TFA})_{1} \\
(\mathrm{SA})_{1} \cdot(\mathrm{DMA})_{1} \cdot(\mathrm{TFA})_{1} \\
(\mathrm{SA})_{1} \cdot(\mathrm{DMA})_{2} \cdot(\mathrm{TFA})_{1}\end{array}$ & $\begin{array}{l}1.03 \times 10^{-5} \\
2.26 \times 10^{-5} \\
1.16 \times 10^{-2} \\
1.91 \times 10^{2}\end{array}$ & $\begin{array}{l}8.75 \times 10^{-6} \\
1.77 \times 10^{-4} \\
1.22 \times 10^{-2} \\
1.77 \times 10^{2}\end{array}$ & $\begin{array}{l}7.35 \times 10^{-6} \\
3.08 \times 10^{-4} \\
1.25 \times 10^{-2} \\
1.66 \times 10^{2}\end{array}$ & $\begin{array}{l}6.28 \times 10^{-6} \\
4.16 \times 10^{-4} \\
1.27 \times 10^{-2} \\
1.57 \times 10^{2}\end{array}$ & $\begin{array}{l}5.48 \times 10^{-6} \\
5.01 \times 10^{-4} \\
1.27 \times 10^{-2} \\
1.49 \times 10^{2}\end{array}$ & $\begin{array}{l}4.86 \times 10^{-6} \\
5.67 \times 10^{-4} \\
1.27 \times 10^{-2} \\
1.43 \times 10^{2}\end{array}$ \\
\hline & \multicolumn{6}{|c|}{$260 \mathrm{~K}$} \\
\hline $\begin{array}{l}(\mathrm{SA})_{1} \cdot(\mathrm{TFA})_{1} \\
(\mathrm{DMA})_{1} \cdot(\mathrm{TFA})_{1} \\
(\mathrm{SA})_{1} \cdot(\mathrm{DMA})_{1} \cdot(\mathrm{TFA})_{1} \\
(\mathrm{SA})_{1} \cdot(\mathrm{DMA})_{2} \cdot(\mathrm{TFA})_{1}\end{array}$ & $\begin{array}{l}1.10 \times 10^{-4} \\
2.48 \times 10^{-4} \\
2.45 \times 10^{-1} \\
1.15 \times 10^{4}\end{array}$ & $\begin{array}{l}9.41 \times 10^{-5} \\
2.86 \times 10^{-3} \\
2.62 \times 10^{-1} \\
1.05 \times 10^{4}\end{array}$ & $\begin{array}{l}8.02 \times 10^{-5} \\
5.08 \times 10^{-3} \\
2.73 \times 10^{-1} \\
9.77 \times 10^{3}\end{array}$ & $\begin{array}{l}6.94 \times 10^{-5} \\
6.87 \times 10^{-3} \\
2.79 \times 10^{-1} \\
9.14 \times 10^{3}\end{array}$ & $\begin{array}{l}6.11 \times 10^{-5} \\
8.28 \times 10^{-3} \\
2.83 \times 10^{-1} \\
8.61 \times 10^{3}\end{array}$ & $\begin{array}{l}5.47 \times 10^{-5} \\
9.36 \times 10^{-3} \\
2.84 \times 10^{-1} \\
8.18 \times 10^{3}\end{array}$ \\
\hline
\end{tabular}


Table S8. Cartesian coordinates of the most stable hydrated clusters in the present study at the M06-2X/6-311++G(3df,3pd) level of theory.

$(\mathrm{SA})_{1} \cdot\left(\mathrm{H}_{2} \mathrm{O}\right)_{1}$

\begin{tabular}{lccc}
\hline Atoms & $\mathrm{X}$ & $\mathrm{Y}$ & $\mathrm{Z}$ \\
\hline $\mathrm{S}$ & & & \\
$\mathrm{O}$ & 0.574277 & -0.075955 & 0.121591 \\
$\mathrm{O}$ & -0.215605 & 0.288808 & 1.248460 \\
$\mathrm{O}$ & 1.737700 & -0.870444 & 0.247437 \\
$\mathrm{O}$ & -0.338533 & -0.732296 & -0.941762 \\
$\mathrm{H}$ & 0.979121 & 1.291265 & -0.552739 \\
$\mathrm{H}$ & -1.289890 & -0.469017 & -0.773800 \\
$\mathrm{O}$ & 1.736148 & 1.150356 & -1.135650 \\
$\mathrm{H}$ & -2.661959 & 0.105985 & -0.109758 \\
$\mathrm{H}$ & -2.259274 & 0.361340 & 0.731812 \\
\hline
\end{tabular}

$(\mathrm{SA})_{1} \cdot\left(\mathrm{H}_{2} \mathrm{O}\right)_{2}$

\begin{tabular}{lccc}
\hline Atoms & $\mathrm{X}$ & $\mathrm{Y}$ & $\mathrm{Z}$ \\
\hline $\mathrm{S}$ & 0.986992 & -0.150388 & -0.099481 \\
$\mathrm{O}$ & 0.432583 & 1.170078 & -0.640315 \\
$\mathrm{O}$ & 1.134685 & 0.126299 & 1.452466 \\
$\mathrm{O}$ & -0.005244 & -1.171702 & -0.181044 \\
$\mathrm{O}$ & 2.277203 & -0.349376 & -0.644188 \\
$\mathrm{H}$ & -0.562404 & 1.301754 & -0.403772 \\
$\mathrm{H}$ & 1.971276 & 0.580502 & 1.615265 \\
$\mathrm{H}$ & -2.423091 & 0.553179 & -0.021326 \\
$\mathrm{O}$ & -2.007743 & 1.446196 & -0.076036 \\
$\mathrm{H}$ & -2.204764 & 1.896542 & 0.747917 \\
$\mathrm{H}$ & -1.846181 & -1.395479 & 0.029141 \\
$\mathrm{O}$ & -2.764155 & -1.093281 & 0.112361 \\
$\mathrm{H}$ & -3.265331 & -1.555999 & -0.561485 \\
\hline
\end{tabular}

$(\mathrm{SA})_{1} \cdot\left(\mathrm{H}_{2} \mathrm{O}\right)_{3}$

\begin{tabular}{lrrr}
\hline Atoms & $\mathrm{X}$ & $\mathrm{Y}$ & $\mathrm{Z}$ \\
\hline $\mathrm{O}$ & & & -1.163177 \\
$\mathrm{H}$ & -1.506990 & -1.371735 & -1.621186 \\
$\mathrm{H}$ & -1.773950 & -0.567008 & -0.314030 \\
$\mathrm{~S}$ & -1.971831 & -1.328300 & 0.161933 \\
$\mathrm{O}$ & 1.244190 & 0.029228 & 0.414141 \\
$\mathrm{O}$ & 2.620912 & 0.184108 & -0.708744 \\
\hline
\end{tabular}




\begin{tabular}{lrrr}
\hline $\mathrm{O}$ & 0.784181 & 1.192212 & -0.765548 \\
$\mathrm{O}$ & 0.322056 & -0.028098 & 1.266286 \\
$\mathrm{H}$ & 0.082261 & -1.372103 & -0.911487 \\
$\mathrm{H}$ & -0.192657 & 1.341878 & -0.695079 \\
$\mathrm{O}$ & -1.876525 & 1.466547 & -0.654087 \\
$\mathrm{H}$ & -2.225041 & 1.049168 & 0.154071 \\
$\mathrm{H}$ & -2.243910 & 2.351570 & -0.702420 \\
$\mathrm{O}$ & -2.325355 & -0.349927 & 1.336061 \\
$\mathrm{H}$ & -2.804143 & -0.522403 & 2.148080 \\
$\mathrm{H}$ & -1.373038 & -0.308106 & 1.551677 \\
\hline
\end{tabular}

$(\mathrm{TFA})_{1} \cdot\left(\mathrm{H}_{2} \mathrm{O}\right)_{1}$

\begin{tabular}{llrr}
\hline Atoms & $\mathrm{X}$ & $\mathrm{Y}$ & $\mathrm{Z}$ \\
\hline $\mathrm{O}$ & 3.362699 & -0.069069 & -0.051778 \\
$\mathrm{H}$ & 3.011637 & 0.832051 & 0.014031 \\
$\mathrm{H}$ & 4.125166 & -0.141963 & 0.530676 \\
$\mathrm{C}$ & 0.333949 & 0.138464 & -0.025218 \\
$\mathrm{O}$ & 0.874293 & 1.217382 & -0.027412 \\
$\mathrm{O}$ & 0.909025 & -1.041694 & -0.032221 \\
$\mathrm{H}$ & 1.895390 & -0.910166 & -0.028620 \\
$\mathrm{C}$ & -1.202835 & -0.003071 & 0.004431 \\
$\mathrm{~F}$ & -1.583079 & -0.615283 & 1.132693 \\
$\mathrm{~F}$ & -1.629468 & -0.733550 & -1.030289 \\
$\mathrm{~F}$ & -1.786010 & 1.188251 & -0.046857 \\
\hline
\end{tabular}

$(\mathrm{TFA})_{1} \cdot\left(\mathrm{H}_{2} \mathrm{O}\right)_{2}$

\begin{tabular}{llcc}
\hline Atoms & $\mathrm{X}$ & $\mathrm{Y}$ & $\mathrm{Z}$ \\
\hline $\mathrm{H}$ & -2.363187 & 1.452463 & -0.054670 \\
$\mathrm{O}$ & -3.313308 & 1.240393 & -0.060936 \\
$\mathrm{H}$ & -3.743089 & 1.802363 & 0.590710 \\
$\mathrm{H}$ & -3.183163 & -0.447357 & 0.032091 \\
$\mathrm{H}$ & -3.222819 & -1.881344 & -0.632125 \\
$\mathrm{O}$ & -2.797748 & -1.356898 & 0.054197 \\
$\mathrm{C}$ & 0.139960 & 0.005991 & 0.000751 \\
$\mathrm{O}$ & -0.523776 & 1.019907 & -0.038579 \\
$\mathrm{O}$ & -0.269253 & -1.224491 & 0.058303 \\
$\mathrm{H}$ & -1.296106 & -1.289418 & 0.057130 \\
$\mathrm{C}$ & 1.683582 & 0.076656 & -0.004439 \\
$\mathrm{~F}$ & 2.182969 & -0.659920 & -1.002578 \\
$\mathrm{~F}$ & 2.171107 & -0.398651 & 1.148131 \\
$\mathrm{~F}$ & 2.101456 & 1.329252 & -0.153874 \\
\hline
\end{tabular}


$(\mathrm{TFA})_{1} \cdot\left(\mathrm{H}_{2} \mathrm{O}\right)_{3}$

\begin{tabular}{lccc}
\hline Atoms & $\mathrm{X}$ & $\mathrm{Y}$ & $\mathrm{Z}$ \\
\hline $\mathrm{H}$ & -1.745831 & 1.668112 & -0.275550 \\
$\mathrm{O}$ & -2.651031 & 2.006936 & -0.351570 \\
$\mathrm{H}$ & -2.625124 & 2.915354 & -0.048644 \\
$\mathrm{H}$ & -2.845443 & -1.289027 & 0.040340 \\
$\mathrm{H}$ & -2.419424 & -2.329993 & -1.044649 \\
$\mathrm{O}$ & -2.135400 & -1.924108 & -0.223709 \\
$\mathrm{C}$ & 0.620692 & -0.087283 & -0.028386 \\
$\mathrm{O}$ & -0.122011 & 0.858538 & -0.042894 \\
$\mathrm{O}$ & 0.324986 & -1.343761 & -0.079687 \\
$\mathrm{H}$ & -0.681481 & -1.514816 & -0.141124 \\
$\mathrm{C}$ & 2.152349 & 0.113896 & 0.056531 \\
$\mathrm{~F}$ & 2.738731 & -0.370895 & -1.034687 \\
$\mathrm{~F}$ & 2.647816 & -0.523804 & 1.112156 \\
$\mathrm{~F}$ & 2.456020 & 1.394967 & 0.160798 \\
$\mathrm{O}$ & -4.028624 & -0.181646 & 0.404797 \\
$\mathrm{H}$ & -4.346195 & -0.139677 & 1.307992 \\
$\mathrm{H}$ & -3.661203 & 0.700298 & 0.192867 \\
\hline
\end{tabular}

$(\mathrm{SA})_{1} \cdot(\mathrm{TFA})_{1} \cdot\left(\mathrm{H}_{2} \mathrm{O}\right)_{1}$

\begin{tabular}{lrrr}
\hline Atoms & X & $\mathrm{Y}$ & $\mathrm{Z}$ \\
\hline $\mathrm{C}$ & 1.740147 & -0.060491 & -0.076518 \\
$\mathrm{O}$ & 1.029422 & 0.615663 & -0.779728 \\
$\mathrm{O}$ & 1.411654 & -1.090282 & 0.633207 \\
$\mathrm{H}$ & 0.439288 & -1.292383 & 0.540432 \\
$\mathrm{C}$ & 3.247734 & 0.254390 & 0.054113 \\
$\mathrm{~F}$ & 3.959442 & -0.755137 & -0.433482 \\
$\mathrm{~F}$ & 3.573254 & 0.421464 & 1.329264 \\
$\mathrm{~F}$ & 3.553181 & 1.350586 & -0.610669 \\
$\mathrm{~S}$ & -2.110935 & -0.516021 & -0.126415 \\
$\mathrm{O}$ & -3.414932 & -0.924559 & -0.511050 \\
$\mathrm{O}$ & -1.154261 & -1.505286 & 0.277396 \\
$\mathrm{O}$ & -2.196242 & 0.497585 & 1.037383 \\
$\mathrm{O}$ & -1.524253 & 0.298837 & -1.299437 \\
$\mathrm{H}$ & -3.050500 & 1.016221 & 0.952365 \\
$\mathrm{H}$ & -0.549434 & 0.458204 & -1.153322 \\
$\mathrm{O}$ & -4.558925 & 1.458392 & 0.547073 \\
$\mathrm{H}$ & -4.776658 & 0.705609 & -0.018806 \\
$\mathrm{H}$ & -4.727621 & 2.250282 & 0.031612 \\
\hline
\end{tabular}


$(\mathrm{SA})_{1} \cdot(\mathrm{TFA})_{1} \cdot\left(\mathrm{H}_{2} \mathrm{O}\right)_{2}$

\begin{tabular}{lrrr}
\hline Atoms & X & Y & \multicolumn{1}{l}{ Z } \\
\hline S & 1.665172 & -0.721455 & -0.012451 \\
$\mathrm{O}$ & 2.985776 & -1.190736 & 0.234451 \\
$\mathrm{O}$ & 0.675877 & -1.682138 & -0.413534 \\
$\mathrm{O}$ & 1.653962 & 0.382581 & -1.068227 \\
$\mathrm{O}$ & 1.195849 & -0.022349 & 1.286405 \\
$\mathrm{H}$ & 2.396454 & 1.092043 & -0.894464 \\
$\mathrm{H}$ & 0.223414 & 0.185551 & 1.218072 \\
$\mathrm{O}$ & 3.483939 & 2.024975 & -0.618749 \\
$\mathrm{H}$ & 4.255516 & 1.521016 & -0.262914 \\
$\mathrm{H}$ & 3.279295 & 2.716191 & 0.015075 \\
$\mathrm{O}$ & 5.286734 & 0.378848 & 0.403672 \\
$\mathrm{H}$ & 4.662400 & -0.361192 & 0.455270 \\
$\mathrm{H}$ & 6.060791 & 0.053152 & -0.058947 \\
$\mathrm{C}$ & -2.113785 & -0.102309 & 0.120697 \\
$\mathrm{O}$ & -1.369774 & 0.442463 & 0.899504 \\
$\mathrm{O}$ & -1.842888 & -1.058407 & -0.705776 \\
$\mathrm{H}$ & -0.882766 & -1.331250 & -0.638928 \\
$\mathrm{C}$ & -3.594951 & 0.326537 & 0.016461 \\
$\mathrm{~F}$ & -4.388484 & -0.712102 & 0.246093 \\
$\mathrm{~F}$ & -3.846442 & 0.785124 & -1.203932 \\
$\mathrm{~F}$ & -3.869655 & 1.273699 & 0.891300 \\
\hline
\end{tabular}

$(\mathrm{SA})_{1} \cdot(\mathrm{TFA})_{1} \cdot\left(\mathrm{H}_{2} \mathrm{O}\right)_{3}$

\begin{tabular}{lrrr}
\hline Atoms & $\mathrm{X}$ & $\mathrm{Y}$ & $\mathrm{Z}$ \\
\hline $\mathrm{S}$ & -1.835354 & 0.418045 & -0.544635 \\
$\mathrm{O}$ & -3.017216 & -0.040423 & -1.196473 \\
$\mathrm{O}$ & -0.659448 & 0.626414 & -1.346608 \\
$\mathrm{O}$ & -1.416089 & -0.556034 & 0.564642 \\
$\mathrm{O}$ & -2.186072 & 1.724279 & 0.173105 \\
$\mathrm{H}$ & -2.229160 & -0.935004 & 1.079103 \\
$\mathrm{H}$ & -1.328371 & 2.207843 & 0.473082 \\
$\mathrm{O}$ & -3.424194 & -1.495170 & 1.746353 \\
$\mathrm{H}$ & -4.174023 & -1.531702 & 1.104608 \\
$\mathrm{H}$ & -3.739818 & -1.024952 & 2.520786 \\
$\mathrm{O}$ & -5.242764 & -1.348471 & -0.177002 \\
$\mathrm{H}$ & -4.641108 & -0.853171 & -0.754547 \\
$\mathrm{H}$ & -5.547167 & -2.103922 & -0.683027 \\
$\mathrm{C}$ & 2.356454 & 0.089545 & -0.161069 \\
$\mathrm{O}$ & 2.085541 & 1.109932 & 0.415460 \\
$\mathrm{O}$ & 1.629739 & -0.574137 & -1.002407 \\
\hline
\end{tabular}




\begin{tabular}{lrrr}
\hline $\mathrm{H}$ & 0.742770 & -0.136287 & -1.139376 \\
$\mathrm{C}$ & 3.710573 & -0.616245 & 0.081107 \\
$\mathrm{~F}$ & 4.276822 & -0.964516 & -1.066093 \\
$\mathrm{~F}$ & 3.513580 & -1.716825 & 0.802414 \\
$\mathrm{~F}$ & 4.538499 & 0.174187 & 0.738743 \\
$\mathrm{O}$ & -0.012733 & 2.816246 & 0.823086 \\
$\mathrm{H}$ & 0.138894 & 3.087922 & 1.730675 \\
$\mathrm{H}$ & 0.727274 & 2.224052 & 0.585800 \\
\hline
\end{tabular}

$(\text { DMA })_{1} \cdot(\mathrm{TFA})_{1} \cdot\left(\mathrm{H}_{2} \mathrm{O}\right)_{1}$

\begin{tabular}{lrrr}
\hline Atoms & X & \multicolumn{1}{l}{$\mathrm{Y}$} \\
\hline $\mathrm{H}$ & -1.361042 & -0.630567 & -0.329075 \\
$\mathrm{O}$ & -2.098890 & 2.282775 & -0.404316 \\
$\mathrm{H}$ & -1.169200 & 2.035559 & -0.205440 \\
$\mathrm{H}$ & -2.071265 & 3.108162 & -0.888350 \\
$\mathrm{C}$ & 0.708817 & 0.107761 & -0.099332 \\
$\mathrm{O}$ & 0.296568 & 1.228835 & 0.197598 \\
$\mathrm{O}$ & 0.058897 & -0.905533 & -0.447083 \\
$\mathrm{C}$ & 2.238137 & -0.139235 & -0.001052 \\
$\mathrm{~F}$ & 2.700021 & -0.747844 & -1.091943 \\
$\mathrm{~F}$ & 2.499831 & -0.930899 & 1.044698 \\
$\mathrm{~F}$ & 2.925415 & 0.982628 & 0.160662 \\
$\mathrm{~N}$ & -2.422381 & -0.416110 & -0.086683 \\
$\mathrm{H}$ & -2.619900 & 0.509038 & -0.488809 \\
$\mathrm{C}$ & -2.485844 & -0.295752 & 1.382678 \\
$\mathrm{H}$ & -2.176460 & -1.241482 & 1.820915 \\
$\mathrm{H}$ & -1.800466 & 0.491317 & 1.684853 \\
$\mathrm{H}$ & -3.498498 & -0.050868 & 1.693989 \\
$\mathrm{O}$ & -3.296421 & -1.460613 & -0.639700 \\
$\mathrm{H}$ & -3.191837 & -1.478234 & -1.720381 \\
$\mathrm{H}$ & -2.990406 & -2.422005 & -0.235096 \\
$\mathrm{H}$ & -4.332398 & -1.264697 & -0.371729 \\
\hline
\end{tabular}

$(\text { DMA })_{1} \cdot(\text { TFA })_{1} \cdot\left(\mathrm{H}_{2} \mathrm{O}\right)_{2}$

\begin{tabular}{lrrr}
\hline Atoms & $\mathrm{X}$ & $\mathrm{Y}$ & $\mathrm{Z}$ \\
\hline $\mathrm{H}$ & -1.006079 & -0.898345 & -0.191929 \\
$\mathrm{O}$ & -3.573128 & 1.093086 & -1.049159 \\
$\mathrm{H}$ & -2.983335 & 1.749976 & -0.620828 \\
$\mathrm{H}$ & -3.684941 & 1.378299 & -1.956978 \\
$\mathrm{C}$ & 1.036992 & 0.066932 & -0.023389 \\
$\mathrm{O}$ & 0.549472 & 1.130317 & 0.359707 \\
\hline
\end{tabular}




\begin{tabular}{lrrr}
\hline $\mathrm{O}$ & 0.462761 & -0.986588 & -0.373269 \\
$\mathrm{C}$ & 2.586245 & -0.021886 & -0.083020 \\
$\mathrm{~F}$ & 2.992559 & -0.316115 & -1.319563 \\
$\mathrm{~F}$ & 3.029174 & -0.987062 & 0.726992 \\
$\mathrm{~F}$ & 3.175201 & 1.109824 & 0.277494 \\
$\mathrm{~N}$ & -2.071647 & -0.998853 & 0.040769 \\
$\mathrm{H}$ & -2.610941 & -0.322880 & -0.524342 \\
$\mathrm{C}$ & -2.254449 & -0.669697 & 1.467865 \\
$\mathrm{H}$ & -1.600418 & -1.308057 & 2.056940 \\
$\mathrm{H}$ & -1.988958 & 0.372115 & 1.621581 \\
$\mathrm{H}$ & -3.291689 & -0.834944 & 1.750581 \\
$\mathrm{C}$ & -2.462569 & -2.378903 & -0.293507 \\
$\mathrm{H}$ & -2.280379 & -2.553158 & -1.349675 \\
$\mathrm{H}$ & -1.852580 & -3.062791 & 0.291183 \\
$\mathrm{H}$ & -3.514698 & -2.533295 & -0.066404 \\
$\mathrm{O}$ & -1.747047 & 2.440993 & 0.353316 \\
$\mathrm{H}$ & -1.551746 & 3.369527 & 0.483594 \\
$\mathrm{H}$ & -0.878897 & 1.974563 & 0.324126 \\
\hline
\end{tabular}

$(\mathrm{SA})_{1} \cdot(\mathrm{DMA})_{1} \cdot(\mathrm{TFA})_{1} \cdot\left(\mathrm{H}_{2} \mathrm{O}\right)_{1}$

\begin{tabular}{|c|c|c|c|}
\hline Atoms & $X$ & $\mathrm{Y}$ & $\mathrm{Z}$ \\
\hline S & 1.034353 & -0.956744 & 0.107501 \\
\hline $\mathrm{O}$ & 2.336821 & -1.554530 & 0.274425 \\
\hline $\mathrm{O}$ & 0.044751 & -1.830524 & -0.500491 \\
\hline $\mathrm{O}$ & 1.090386 & 0.344758 & -0.562713 \\
\hline $\mathrm{O}$ & 0.558809 & -0.674037 & 1.576180 \\
\hline $\mathrm{H}$ & 2.553330 & 0.931518 & -0.430724 \\
\hline $\mathrm{H}$ & -0.375006 & -0.361564 & 1.537260 \\
\hline $\mathrm{C}$ & -2.608037 & -0.122899 & 0.088614 \\
\hline $\mathrm{O}$ & -1.975618 & 0.136444 & 1.083232 \\
\hline $\mathrm{O}$ & -2.275143 & -0.876389 & -0.903212 \\
\hline $\mathrm{H}$ & -1.341506 & -1.269827 & -0.774286 \\
\hline $\mathrm{C}$ & -4.020699 & 0.472166 & -0.112886 \\
\hline $\mathrm{F}$ & -4.927411 & -0.500535 & -0.131919 \\
\hline $\mathrm{F}$ & -4.090005 & 1.126855 & -1.267606 \\
\hline $\mathrm{F}$ & -4.325594 & 1.309474 & 0.861730 \\
\hline $\mathrm{N}$ & 3.541588 & 1.248997 & -0.200254 \\
\hline $\mathrm{H}$ & 4.161830 & 0.512597 & -0.579043 \\
\hline $\mathrm{C}$ & 3.662081 & 1.246204 & 1.275086 \\
\hline $\mathrm{H}$ & 2.933885 & 1.941513 & 1.684081 \\
\hline $\mathrm{H}$ & 3.449698 & 0.241897 & 1.629588 \\
\hline $\mathrm{H}$ & 4.668870 & 1.544548 & 1.554988 \\
\hline $\mathrm{C}$ & 3.813993 & 2.558426 & -0.818454 \\
\hline $\mathrm{H}$ & 3.695366 & 2.476022 & -1.894451 \\
\hline
\end{tabular}




\begin{tabular}{lrrr}
\hline $\mathrm{H}$ & 3.104814 & 3.283870 & -0.429339 \\
$\mathrm{H}$ & 4.827229 & 2.870544 & -0.578944 \\
$\mathrm{O}$ & 4.823467 & -1.137414 & -0.709143 \\
$\mathrm{H}$ & 5.174213 & -1.672463 & -1.422028 \\
$\mathrm{H}$ & 3.981803 & -1.545702 & -0.435565 \\
\hline
\end{tabular}

$(\mathrm{SA})_{1} \cdot(\mathrm{DMA})_{1} \cdot(\mathrm{TFA})_{1} \cdot\left(\mathrm{H}_{2} \mathrm{O}\right)_{2}$

\begin{tabular}{|c|c|c|c|}
\hline Atoms & $X$ & $\mathrm{Y}$ & $\mathrm{Z}$ \\
\hline $\mathrm{H}$ & -0.080007 & -0.419317 & 1.240587 \\
\hline $\mathrm{S}$ & -2.015823 & -0.670788 & -0.135703 \\
\hline $\mathrm{O}$ & -3.090703 & 0.293975 & -0.159460 \\
\hline $\mathrm{O}$ & -1.421949 & -0.796430 & 1.219289 \\
\hline $\mathrm{O}$ & -0.963591 & -0.410056 & -1.108430 \\
\hline $\mathrm{O}$ & -2.659618 & -2.036520 & -0.458734 \\
\hline $\mathrm{H}$ & -0.219115 & 1.243525 & -0.650434 \\
\hline $\mathrm{H}$ & -1.944966 & -2.744951 & -0.409905 \\
\hline $\mathrm{N}$ & -0.431023 & 2.220308 & -0.389006 \\
\hline $\mathrm{H}$ & -0.985086 & 2.154117 & 0.497300 \\
\hline $\mathrm{O}$ & -2.211616 & 2.118407 & 1.703286 \\
\hline $\mathrm{H}$ & -2.777708 & 1.503098 & 1.199760 \\
\hline $\mathrm{H}$ & -1.962752 & 1.618067 & 2.485390 \\
\hline $\mathrm{O}$ & -0.657324 & -3.667654 & -0.274406 \\
\hline $\mathrm{H}$ & -0.337722 & -3.983011 & -1.123021 \\
\hline $\mathrm{H}$ & 0.060250 & -3.109994 & 0.072353 \\
\hline $\mathrm{C}$ & 1.680442 & -0.836609 & 0.511218 \\
\hline $\mathrm{O}$ & 1.525004 & -2.008004 & 0.298224 \\
\hline $\mathrm{O}$ & 0.895257 & -0.010079 & 1.122044 \\
\hline $\mathrm{C}$ & 2.934497 & -0.107072 & -0.020026 \\
\hline $\mathrm{F}$ & 3.475713 & 0.678497 & 0.907292 \\
\hline $\mathrm{F}$ & 2.566594 & 0.679169 & -1.044104 \\
\hline $\mathrm{F}$ & 3.851497 & -0.945849 & -0.451329 \\
\hline $\mathrm{C}$ & 0.793544 & 3.010180 & -0.152221 \\
\hline $\mathrm{H}$ & 1.381867 & 2.517530 & 0.615449 \\
\hline $\mathrm{H}$ & 1.365009 & 3.068380 & -1.073802 \\
\hline $\mathrm{H}$ & 0.505829 & 4.006536 & 0.171968 \\
\hline $\mathrm{C}$ & -1.324994 & 2.775882 & -1.431283 \\
\hline $\mathrm{H}$ & -0.776761 & 2.840975 & -2.366849 \\
\hline $\mathrm{H}$ & -2.171916 & 2.103519 & -1.534325 \\
\hline $\mathrm{H}$ & -1.655435 & 3.762230 & -1.117548 \\
\hline
\end{tabular}


$(\mathrm{SA})_{1} \cdot(\mathrm{DMA})_{2} \cdot(\mathrm{TFA})_{1} \cdot\left(\mathrm{H}_{2} \mathrm{O}\right)_{1}$

\begin{tabular}{|c|c|c|c|}
\hline Atoms & $\mathrm{X}$ & $Y$ & $\mathrm{Z}$ \\
\hline$S$ & 1.317575 & -1.578670 & 0.034019 \\
\hline $\mathrm{O}$ & 0.602600 & -2.827653 & -0.209331 \\
\hline $\mathrm{O}$ & 2.729985 & -1.753850 & 0.289467 \\
\hline $\mathrm{O}$ & 0.631230 & -0.747733 & 1.013227 \\
\hline $\mathrm{O}$ & 1.279068 & -0.754265 & -1.316709 \\
\hline $\mathrm{H}$ & -0.981677 & -2.508475 & 0.023221 \\
\hline $\mathrm{C}$ & -1.119567 & 1.291251 & -0.575722 \\
\hline $\mathrm{O}$ & -0.178033 & 2.021477 & -0.301340 \\
\hline $\mathrm{O}$ & -1.141006 & 0.215848 & -1.225427 \\
\hline $\mathrm{H}$ & 0.362202 & -0.351713 & -1.411671 \\
\hline $\mathrm{C}$ & -2.527188 & 1.723191 & -0.076991 \\
\hline $\mathrm{F}$ & -3.188461 & 0.662345 & 0.431858 \\
\hline $\mathrm{F}$ & -3.263536 & 2.191470 & -1.083601 \\
\hline $\mathrm{F}$ & -2.484914 & 2.648185 & 0.866889 \\
\hline $\mathrm{N}$ & -1.927133 & -2.059672 & 0.164883 \\
\hline $\mathrm{H}$ & -1.782659 & -1.102421 & -0.207388 \\
\hline $\mathrm{C}$ & -2.228207 & -2.015950 & 1.609083 \\
\hline $\mathrm{H}$ & -2.284308 & -3.034702 & 1.984076 \\
\hline $\mathrm{H}$ & -1.421423 & -1.480650 & 2.099856 \\
\hline $\mathrm{H}$ & -3.176448 & -1.508831 & 1.762474 \\
\hline $\mathrm{C}$ & -2.955880 & -2.730413 & -0.648409 \\
\hline $\mathrm{H}$ & -2.647289 & -2.707314 & -1.689073 \\
\hline $\mathrm{H}$ & -3.061008 & -3.759431 & -0.315227 \\
\hline $\mathrm{H}$ & -3.900081 & -2.204393 & -0.532557 \\
\hline $\mathrm{H}$ & 1.449604 & 1.270086 & 0.280764 \\
\hline $\mathrm{N}$ & 2.448932 & 1.511785 & 0.378972 \\
\hline $\mathrm{H}$ & 2.983863 & 0.914422 & -0.283666 \\
\hline $\mathrm{C}$ & 2.916511 & 1.223035 & 1.751289 \\
\hline $\mathrm{H}$ & 2.329844 & 1.812998 & 2.450027 \\
\hline $\mathrm{H}$ & 2.777870 & 0.164372 & 1.941559 \\
\hline $\mathrm{H}$ & 3.967707 & 1.490841 & 1.820894 \\
\hline $\mathrm{C}$ & 2.610701 & 2.923039 & -0.022036 \\
\hline $\mathrm{H}$ & 2.211958 & 3.043982 & -1.023493 \\
\hline $\mathrm{H}$ & 2.044866 & 3.548317 & 0.662101 \\
\hline $\mathrm{H}$ & 3.666637 & 3.177723 & 0.005951 \\
\hline $\mathrm{O}$ & 4.220637 & -0.000046 & -1.160360 \\
\hline $\mathrm{H}$ & 3.948293 & -0.103261 & -2.075381 \\
\hline $\mathrm{H}$ & 3.876384 & -0.800751 & -0.719571 \\
\hline
\end{tabular}


$(\mathrm{SA})_{1} \cdot(\mathrm{DMA})_{2} \cdot(\mathrm{TFA})_{1} \cdot\left(\mathrm{H}_{2} \mathrm{O}\right)_{2}$

\begin{tabular}{|c|c|c|c|}
\hline Atoms & $\mathrm{X}$ & $\mathrm{Y}$ & $\mathrm{Z}$ \\
\hline$S$ & 1.446453 & 1.638159 & -0.268362 \\
\hline $\mathrm{O}$ & 1.240698 & 0.838327 & 0.940887 \\
\hline $\mathrm{O}$ & 2.769707 & 2.205594 & -0.357425 \\
\hline $\mathrm{O}$ & 0.378835 & 2.609877 & -0.469197 \\
\hline $\mathrm{O}$ & 1.365517 & 0.620200 & -1.463670 \\
\hline $\mathrm{H}$ & -1.139022 & 2.152579 & -0.190917 \\
\hline $\mathrm{H}$ & 0.422298 & 0.238972 & -1.480437 \\
\hline $\mathrm{C}$ & -1.033881 & -1.281006 & -0.512056 \\
\hline $\mathrm{O}$ & -1.026730 & -0.268888 & -1.241654 \\
\hline $\mathrm{O}$ & -0.095892 & -1.946558 & -0.075005 \\
\hline $\mathrm{H}$ & 1.630107 & -1.214036 & 0.049678 \\
\hline $\mathrm{C}$ & -2.455999 & -1.777552 & -0.123916 \\
\hline $\mathrm{F}$ & -2.467174 & -2.405864 & 1.051152 \\
\hline $\mathrm{F}$ & -2.921362 & -2.625047 & -1.040387 \\
\hline $\mathrm{F}$ & -3.332415 & -0.765977 & -0.037499 \\
\hline $\mathrm{N}$ & 2.586399 & -1.590610 & 0.130913 \\
\hline $\mathrm{H}$ & 3.243170 & -0.802590 & 0.308761 \\
\hline $\mathrm{C}$ & 2.924339 & -2.241782 & -1.148507 \\
\hline $\mathrm{H}$ & 2.187122 & -3.016133 & -1.342339 \\
\hline $\mathrm{H}$ & 2.887426 & -1.488354 & -1.929165 \\
\hline $\mathrm{H}$ & 3.921695 & -2.667879 & -1.079425 \\
\hline $\mathrm{C}$ & 2.594309 & -2.504002 & 1.287419 \\
\hline $\mathrm{H}$ & 2.330340 & -1.933920 & 2.173611 \\
\hline $\mathrm{H}$ & 1.854380 & -3.280720 & 1.118133 \\
\hline $\mathrm{H}$ & 3.586184 & -2.934221 & 1.399321 \\
\hline $\mathrm{N}$ & -2.124595 & 2.001937 & 0.156991 \\
\hline $\mathrm{H}$ & -2.113395 & 1.059611 & 0.567517 \\
\hline $\mathrm{C}$ & -3.076734 & 2.083548 & -0.965793 \\
\hline $\mathrm{H}$ & -3.002113 & 3.071775 & -1.411934 \\
\hline $\mathrm{H}$ & -2.811448 & 1.317578 & -1.686267 \\
\hline $\mathrm{H}$ & -4.084370 & 1.917131 & -0.593462 \\
\hline $\mathrm{C}$ & -2.363030 & 2.982652 & 1.232989 \\
\hline $\mathrm{H}$ & -1.617803 & 2.825923 & 2.006326 \\
\hline $\mathrm{H}$ & -2.266097 & 3.983181 & 0.820703 \\
\hline $\mathrm{H}$ & -3.359606 & 2.837601 & 1.641675 \\
\hline $\mathrm{O}$ & 4.517788 & 0.356294 & 0.466714 \\
\hline $\mathrm{H}$ & 4.834071 & 0.600201 & 1.338336 \\
\hline $\mathrm{H}$ & 3.997135 & 1.131068 & 0.154912 \\
\hline $\mathrm{O}$ & -1.201170 & 0.114132 & 1.971966 \\
\hline $\mathrm{H}$ & -1.221325 & -0.822975 & 2.180295 \\
\hline $\mathrm{H}$ & -0.290100 & 0.304388 & 1.672010 \\
\hline
\end{tabular}


Table S9. Predicted concentrations of DMA (molecules $\mathrm{cm}^{-3}$ ) by the global chemistry-transport model, temperatures $(T, \mathrm{~K})$, particle formation rates of SA-DMA-TFA system $\left(J_{\text {SA-DMA-TFA }} \mathrm{cm}^{-3}\right.$ $\left.\mathrm{s}^{-1}\right)$ and SA-DMA system $\left(J_{\text {SA-DMA }}, \mathrm{cm}^{-3} \mathrm{~s}^{-1}\right)$ and the enhancement $\left(J_{\text {SA-DMA-TFA }} / J_{\text {SA-DMA }}\right)$ on particle formation rates by TFA.

\begin{tabular}{|c|c|c|c|c|c|c|}
\hline Cities & Month & $\begin{array}{c}\text { [DMA] } \\
\left(\text { molecules } \mathrm{cm}^{-3}\right)\end{array}$ & $T(\mathrm{~K})$ & $\begin{array}{c}J_{\text {SA-DMA-TFA }} \\
\left(\mathrm{cm}^{-3} \mathrm{~s}^{-1}\right)\end{array}$ & $\begin{array}{l}J_{\text {SA-DMA }} \\
\left(\mathrm{cm}^{-3} \mathrm{~s}^{-1}\right)\end{array}$ & Enhancement \\
\hline \multirow[t]{12}{*}{ Beijing } & 1 & $9.37 \times 10^{7}$ & 265 & $5.10 \times 10^{2}$ & $3.84 \times 10^{1}$ & 13.27 \\
\hline & 2 & $8.63 \times 10^{7}$ & 269 & $2.99 \times 10^{2}$ & $3.85 \times 10^{1}$ & 7.75 \\
\hline & 3 & $7.49 \times 10^{7}$ & 275 & $1.28 \times 10^{2}$ & $3.79 \times 10^{1}$ & 3.39 \\
\hline & 4 & $6.26 \times 10^{7}$ & 284 & $5.01 \times 10^{1}$ & $3.39 \times 10^{1}$ & 1.48 \\
\hline & 5 & $4.25 \times 10^{7}$ & 291 & $2.29 \times 10^{1}$ & $2.11 \times 10^{1}$ & 1.08 \\
\hline & 6 & $4.45 \times 10^{7}$ & 296 & $1.56 \times 10^{1}$ & $1.51 \times 10^{1}$ & 1.03 \\
\hline & 7 & $3.09 \times 10^{7}$ & 298 & 8.30 & 8.19 & 1.01 \\
\hline & 8 & $5.12 \times 10^{7}$ & 296 & $1.78 \times 10^{1}$ & $1.71 \times 10^{1}$ & 1.04 \\
\hline & 9 & $6.01 \times 10^{7}$ & 291 & $3.04 \times 10^{1}$ & $2.67 \times 10^{1}$ & 1.14 \\
\hline & 10 & $7.26 \times 10^{7}$ & 283 & $6.10 \times 10^{1}$ & $3.62 \times 10^{1}$ & 1.69 \\
\hline & 11 & $1.52 \times 10^{8}$ & 274 & $3.22 \times 10^{2}$ & $4.28 \times 10^{1}$ & 7.53 \\
\hline & 12 & $1.04 \times 10^{8}$ & 267 & $4.67 \times 10^{2}$ & $3.95 \times 10^{1}$ & 11.85 \\
\hline \multirow[t]{12}{*}{ Shanghai } & 1 & $5.21 \times 10^{7}$ & 281 & $5.29 \times 10^{1}$ & $3.29 \times 10^{1}$ & 1.61 \\
\hline & 2 & $5.28 \times 10^{7}$ & 282 & $4.96 \times 10^{1}$ & $3.27 \times 10^{1}$ & 1.52 \\
\hline & 3 & $2.56 \times 10^{7}$ & 284 & $2.30 \times 10^{1}$ & $2.02 \times 10^{1}$ & 1.14 \\
\hline & 4 & $2.13 \times 10^{7}$ & 287 & $1.56 \times 10^{1}$ & $1.47 \times 10^{1}$ & 1.06 \\
\hline & 5 & $2.10 \times 10^{7}$ & 291 & $1.10 \times 10^{1}$ & $1.06 \times 10^{1}$ & 1.03 \\
\hline & 6 & $2.51 \times 10^{7}$ & 295 & 9.14 & 8.98 & 1.02 \\
\hline & 7 & $2.16 \times 10^{7}$ & 299 & 4.48 & 4.45 & 1.01 \\
\hline & 8 & $2.49 \times 10^{7}$ & 299 & 5.51 & 5.47 & 1.01 \\
\hline & 9 & $2.89 \times 10^{7}$ & 296 & 9.66 & 9.49 & 1.02 \\
\hline & 10 & $3.58 \times 10^{7}$ & 290 & $2.12 \times 10^{1}$ & $1.97 \times 10^{1}$ & 1.08 \\
\hline & 11 & $6.07 \times 10^{7}$ & 285 & $4.58 \times 10^{1}$ & $3.28 \times 10^{1}$ & 1.40 \\
\hline & 12 & $6.30 \times 10^{7}$ & 280 & $6.82 \times 10^{1}$ & $3.55 \times 10^{1}$ & 1.92 \\
\hline \multirow[t]{12}{*}{ Los Angeles } & 1 & $2.87 \times 10^{7}$ & 275 & $4.33 \times 10^{1}$ & $2.61 \times 10^{1}$ & 1.66 \\
\hline & 2 & $2.99 \times 10^{7}$ & 277 & $4.00 \times 10^{1}$ & $2.63 \times 10^{1}$ & 1.52 \\
\hline & 3 & $2.66 \times 10^{7}$ & 282 & $2.67 \times 10^{1}$ & $2.23 \times 10^{1}$ & 1.20 \\
\hline & 4 & $4.70 \times 10^{7}$ & 288 & $3.07 \times 10^{1}$ & $2.64 \times 10^{1}$ & 1.16 \\
\hline & 5 & $2.49 \times 10^{7}$ & 294 & $1.00 \times 10^{1}$ & 9.83 & 1.02 \\
\hline & 6 & $6.42 \times 10^{7}$ & 298 & $1.82 \times 10^{1}$ & $1.76 \times 10^{1}$ & 1.04 \\
\hline & 7 & $2.81 \times 10^{7}$ & 301 & 4.98 & 4.95 & 1.01 \\
\hline & 8 & $4.36 \times 10^{7}$ & 301 & 8.81 & 8.72 & 1.01 \\
\hline & 9 & $2.80 \times 10^{7}$ & 296 & 9.31 & 9.15 & 1.02 \\
\hline & 10 & $4.16 \times 10^{7}$ & 291 & $2.25 \times 10^{1}$ & $2.08 \times 10^{1}$ & 1.08 \\
\hline & 11 & $3.56 \times 10^{7}$ & 284 & $3.12 \times 10^{1}$ & $2.56 \times 10^{1}$ & 1.22 \\
\hline & 12 & $7.14 \times 10^{7}$ & 278 & $9.23 \times 10^{1}$ & $3.73 \times 10^{1}$ & 2.48 \\
\hline \multirow[t]{3}{*}{ New Delhi } & 1 & $1.42 \times 10^{8}$ & 287 & $7.70 \times 10^{1}$ & $4.12 \times 10^{1}$ & 1.87 \\
\hline & 2 & $1.36 \times 10^{8}$ & 291 & $5.43 \times 10^{1}$ & $3.82 \times 10^{1}$ & 1.42 \\
\hline & 3 & $8.49 \times 10^{7}$ & 297 & $2.52 \times 10^{1}$ & $2.36 \times 10^{1}$ & 1.07 \\
\hline
\end{tabular}




\begin{tabular}{cccccc}
\hline 4 & $9.66 \times 10^{7}$ & 304 & $1.46 \times 10^{1}$ & $1.44 \times 10^{1}$ & 1.02 \\
5 & $8.40 \times 10^{7}$ & 308 & 7.85 & 7.81 & 1.01 \\
6 & $6.04 \times 10^{7}$ & 309 & 4.56 & 4.54 & 1.00 \\
7 & $6.15 \times 10^{7}$ & 306 & 7.05 & 7.01 & 1.01 \\
8 & $6.08 \times 10^{7}$ & 303 & $1.01 \times 10^{1}$ & $1.00 \times 10^{1}$ & 1.01 \\
9 & $5.75 \times 10^{7}$ & 301 & $1.20 \times 10^{1}$ & $1.19 \times 10^{1}$ & 1.02 \\
10 & $9.52 \times 10^{7}$ & 298 & $2.52 \times 10^{1}$ & $2.37 \times 10^{1}$ & 1.07 \\
11 & $1.15 \times 10^{8}$ & 293 & $4.18 \times 10^{1}$ & $3.40 \times 10^{1}$ & 1.23 \\
12 & $1.24 \times 10^{8}$ & 289 & $5.94 \times 10^{1}$ & $3.87 \times 10^{1}$ & 1.53 \\
\hline
\end{tabular}


Table S10. Gibbs free formation energies $(\Delta G, \mathrm{kcal} / \mathrm{mol})$ of clusters at different temperatures in different months of the studied cities shown in Table S9.

\begin{tabular}{|c|c|c|c|c|c|}
\hline \multirow{2}{*}{ Clusters } & \multicolumn{5}{|c|}{$\Delta G(\mathrm{kcal} / \mathrm{mol})$} \\
\hline & $265 \mathrm{~K}$ & $267 \mathrm{~K}$ & $269 \mathrm{~K}$ & $270 \mathrm{~K}$ & $274 \mathrm{~K}$ \\
\hline$(\mathrm{SA})_{1} \cdot(\mathrm{DMA})_{1}$ & -15.80 & -15.73 & -15.66 & -15.63 & -15.49 \\
\hline$(\mathrm{SA})_{1} \cdot(\mathrm{DMA})_{1} \cdot(\mathrm{TFA})_{1}$ & -28.30 & -28.16 & -28.01 & -27.94 & -27.65 \\
\hline$(\mathrm{SA})_{1} \cdot(\mathrm{DMA})_{2} \cdot(\mathrm{TFA})_{1}$ & -38.01 & -46.45 & -46.21 & -46.09 & -45.62 \\
\hline$(\mathrm{SA})_{1} \cdot(\mathrm{TFA})_{1}$ & -8.35 & -8.28 & -8.21 & -8.18 & -8.04 \\
\hline$(\mathrm{SA})_{1} \cdot(\mathrm{DMA})_{1} \cdot(\mathrm{TFA})_{2}$ & -38.01 & -37.78 & -37.56 & -37.44 & -36.99 \\
\hline$(\mathrm{SA})_{1} \cdot(\mathrm{DMA})_{2} \cdot(\mathrm{TFA})_{2}$ & -54.91 & -54.57 & -54.24 & -54.07 & -53.41 \\
\hline$(\mathrm{SA})_{1} \cdot(\mathrm{DMA})_{3} \cdot(\mathrm{TFA})_{2}$ & -71.48 & -71.07 & -70.65 & -70.45 & -69.62 \\
\hline$(\mathrm{SA})_{1} \cdot(\mathrm{TFA})_{2}$ & -14.65 & -14.49 & -14.34 & -14.26 & -13.95 \\
\hline$(\mathrm{DMA})_{1} \cdot(\mathrm{TFA})_{1}$ & -9.15 & -9.08 & -9.02 & -8.98 & -8.85 \\
\hline$(\mathrm{SA})_{2} \cdot(\mathrm{DMA})_{1}$ & -37.47 & -37.31 & -37.16 & -37.08 & -36.77 \\
\hline$(\mathrm{SA})_{2} \cdot(\mathrm{DMA})_{1} \cdot(\mathrm{TFA})_{1}$ & -43.89 & -43.65 & -43.42 & -43.30 & -42.83 \\
\hline$(\mathrm{SA})_{2} \cdot(\mathrm{DMA})_{2} \cdot(\mathrm{TFA})_{1}$ & -64.41 & -64.08 & -63.74 & -63.58 & -62.91 \\
\hline$(\mathrm{SA})_{2} \cdot(\mathrm{DMA})_{3} \cdot(\mathrm{TFA})_{1}$ & -82.61 & -82.19 & -81.78 & -81.57 & -80.75 \\
\hline$(\mathrm{SA})_{2} \cdot(\mathrm{TFA})_{1}$ & -15.87 & -15.71 & -15.55 & -15.47 & -15.15 \\
\hline$(\mathrm{SA})_{2} \cdot(\mathrm{DMA})_{2}$ & -58.68 & -58.45 & -58.22 & -58.11 & -57.64 \\
\hline$(\mathrm{SA})_{2}$ & -9.55 & -9.48 & -9.41 & -9.38 & -9.24 \\
\hline$(\mathrm{DMA})_{1} \cdot(\mathrm{TFA})_{2}$ & -20.19 & -20.04 & -19.89 & -19.82 & -19.52 \\
\hline$(\mathrm{DMA})_{2} \cdot(\mathrm{TFA})_{2}$ & -36.29 & -36.06 & -35.83 & -35.71 & -35.25 \\
\hline$(\mathrm{TFA})_{2}$ & -6.32 & -6.25 & -6.17 & -6.14 & -5.99 \\
\hline$(\mathrm{SA})_{3} \cdot(\mathrm{DMA})_{1}$ & -51.84 & -51.61 & -51.37 & -51.25 & -50.77 \\
\hline$(\mathrm{SA})_{3} \cdot(\mathrm{DMA})_{2}$ & -76.12 & -75.81 & -75.49 & -75.34 & -74.71 \\
\hline$(\mathrm{SA})_{3} \cdot(\mathrm{DMA})_{3}$ & -97.19 & -96.80 & -96.40 & -96.20 & -95.42 \\
\hline$(\mathrm{SA})_{3}$ & -16.48 & -16.33 & -16.17 & -16.09 & -15.79 \\
\hline$(\mathrm{DMA})_{1} \cdot(\mathrm{TFA})_{3}$ & -24.38 & -24.12 & -23.85 & -23.72 & -23.19 \\
\hline$(\mathrm{DMA})_{2} \cdot(\mathrm{TFA})_{3}$ & -45.59 & -45.27 & -44.93 & -44.77 & -44.11 \\
\hline$(\mathrm{DMA})_{3} \cdot(\mathrm{TFA})_{3}$ & -57.27 & -56.86 & -56.45 & -56.24 & -55.41 \\
\hline$(\mathrm{TFA})_{3}$ & -4.88 & -4.73 & -4.59 & -4.52 & -4.23 \\
\hline
\end{tabular}


Table S10. Continued: Gibbs free formation energies $(\Delta G, \mathrm{kcal} / \mathrm{mol})$ of clusters at different temperatures in different months of the studied cities shown in Table S9.

\begin{tabular}{|c|c|c|c|c|c|}
\hline \multirow{2}{*}{ Clusters } & \multicolumn{5}{|c|}{$\Delta G(\mathrm{kcal} / \mathrm{mol})$} \\
\hline & $275 \mathrm{~K}$ & $277 \mathrm{~K}$ & $278 \mathrm{~K}$ & $280 \mathrm{~K}$ & $281 \mathrm{~K}$ \\
\hline$(\mathrm{SA})_{1} \cdot(\mathrm{DMA})_{1}$ & -15.46 & -15.39 & -15.35 & -15.28 & -15.25 \\
\hline$(\mathrm{SA})_{1} \cdot(\mathrm{DMA})_{1} \cdot(\mathrm{TFA})_{1}$ & -27.57 & -27.43 & -27.36 & -27.21 & -27.14 \\
\hline$(\mathrm{SA})_{1} \cdot(\mathrm{DMA})_{2} \cdot(\mathrm{TFA})_{1}$ & -45.51 & -45.27 & -45.15 & -44.92 & -44.80 \\
\hline$(\mathrm{SA})_{1} \cdot(\mathrm{TFA})_{1}$ & -8.00 & -7.93 & -7.90 & -7.83 & -7.79 \\
\hline$(\mathrm{SA})_{1} \cdot(\mathrm{DMA})_{1} \cdot(\mathrm{TFA})_{2}$ & -36.88 & -36.66 & -36.54 & -36.32 & -36.21 \\
\hline$(\mathrm{SA})_{1} \cdot(\mathrm{DMA})_{2} \cdot(\mathrm{TFA})_{2}$ & -53.24 & -52.91 & -52.74 & -52.41 & -52.24 \\
\hline$(\mathrm{SA})_{1} \cdot(\mathrm{DMA})_{3} \cdot(\mathrm{TFA})_{2}$ & -69.42 & -69.01 & -68.80 & -68.39 & -68.18 \\
\hline$(\mathrm{SA})_{1} \cdot(\mathrm{TFA})_{2}$ & -13.88 & -13.72 & -13.65 & -13.49 & -13.41 \\
\hline$(\mathrm{DMA})_{1} \cdot(\mathrm{TFA})_{1}$ & -8.82 & -8.75 & -8.72 & -8.65 & -8.62 \\
\hline$(\mathrm{SA})_{2} \cdot(\mathrm{DMA})_{1}$ & -36.69 & -36.54 & -36.46 & -36.30 & -36.22 \\
\hline$(\mathrm{SA})_{2} \cdot(\mathrm{DMA})_{1} \cdot(\mathrm{TFA})_{1}$ & -42.72 & -42.48 & -42.37 & -42.13 & -42.01 \\
\hline$(\mathrm{SA})_{2} \cdot(\mathrm{DMA})_{2} \cdot(\mathrm{TFA})_{1}$ & -62.74 & -62.41 & -62.24 & -61.91 & -61.74 \\
\hline$(\mathrm{SA})_{2} \cdot(\mathrm{DMA})_{3} \cdot(\mathrm{TFA})_{1}$ & -80.55 & -80.13 & -79.93 & -79.52 & -79.31 \\
\hline$(\mathrm{SA})_{2} \cdot(\mathrm{TFA})_{1}$ & -15.08 & -14.92 & -14.84 & -14.68 & -14.60 \\
\hline$(\mathrm{SA})_{2} \cdot(\mathrm{DMA})_{2}$ & -57.53 & -57.30 & -57.18 & -56.95 & -56.84 \\
\hline$(\mathrm{SA})_{2}$ & -9.21 & -9.14 & -9.11 & -9.04 & -9.01 \\
\hline$(\mathrm{DMA})_{1} \cdot(\mathrm{TFA})_{2}$ & -19.44 & -19.29 & -19.21 & -19.06 & -18.99 \\
\hline$(\mathrm{DMA})_{2} \cdot(\mathrm{TFA})_{2}$ & -35.13 & -34.90 & -34.79 & -34.55 & -34.44 \\
\hline$(\mathrm{TFA})_{2}$ & -5.95 & -5.87 & -5.84 & -5.76 & -5.72 \\
\hline$(\mathrm{SA})_{3} \cdot(\mathrm{DMA})_{1}$ & -50.65 & -50.41 & -50.30 & -50.06 & -49.94 \\
\hline$(\mathrm{SA})_{3} \cdot(\mathrm{DMA})_{2}$ & -74.56 & -74.25 & -74.09 & -73.78 & -73.62 \\
\hline$(\mathrm{SA})_{3} \cdot(\mathrm{DMA})_{3}$ & -95.22 & -94.83 & -94.63 & -94.24 & -94.04 \\
\hline$(\mathrm{SA})_{3}$ & -15.71 & -15.56 & -15.48 & -15.33 & -15.25 \\
\hline$(\mathrm{DMA})_{1} \cdot(\mathrm{TFA})_{3}$ & -23.05 & -22.79 & -22.65 & -22.39 & -22.25 \\
\hline$(\mathrm{DMA})_{2} \cdot(\mathrm{TFA})_{3}$ & -43.95 & -43.62 & -43.46 & -43.13 & -42.96 \\
\hline$(\mathrm{DMA})_{3} \cdot(\mathrm{TFA})_{3}$ & -55.21 & -54.80 & -54.59 & -54.18 & -53.97 \\
\hline$(\mathrm{TFA})_{3}$ & -4.15 & -4.01 & -3.93 & -3.79 & -3.72 \\
\hline
\end{tabular}


Table S10. Continued: Gibbs free formation energies $(\Delta G, \mathrm{kcal} / \mathrm{mol})$ of clusters at different temperatures in different months of the studied cities shown in Table S9.

\begin{tabular}{|c|c|c|c|c|c|}
\hline \multirow{2}{*}{ Clusters } & \multicolumn{5}{|c|}{$\Delta G(\mathrm{kcal} / \mathrm{mol})$} \\
\hline & $282 \mathrm{~K}$ & $283 \mathrm{~K}$ & $284 \mathrm{~K}$ & $285 \mathrm{~K}$ & $286 \mathrm{~K}$ \\
\hline$(\mathrm{SA})_{1} \cdot(\mathrm{DMA})_{1}$ & -15.22 & -15.18 & -15.15 & -15.11 & -15.08 \\
\hline$(\mathrm{SA})_{1} \cdot(\mathrm{DMA})_{1} \cdot(\mathrm{TFA})_{1}$ & -27.06 & -26.99 & -26.92 & -26.85 & -26.77 \\
\hline$(\mathrm{SA})_{1} \cdot(\mathrm{DMA})_{2} \cdot(\mathrm{TFA})_{1}$ & -44.68 & -44.57 & -44.45 & -44.33 & -44.22 \\
\hline$(\mathrm{SA})_{1} \cdot(\mathrm{TFA})_{1}$ & -7.76 & -7.72 & -7.69 & -7.65 & -7.62 \\
\hline$(\mathrm{SA})_{1} \cdot(\mathrm{DMA})_{1} \cdot(\mathrm{TFA})_{2}$ & -36.09 & -35.98 & -35.87 & -35.76 & -35.64 \\
\hline$(\mathrm{SA})_{1} \cdot(\mathrm{DMA})_{2} \cdot(\mathrm{TFA})_{2}$ & -52.07 & -51.91 & -51.74 & -51.57 & -51.41 \\
\hline$(\mathrm{SA})_{1} \cdot(\mathrm{DMA})_{3} \cdot(\mathrm{TFA})_{2}$ & -67.98 & -67.77 & -67.57 & -67.36 & -67.15 \\
\hline$(\mathrm{SA})_{1} \cdot(\mathrm{TFA})_{2}$ & -13.34 & -13.26 & -13.18 & -13.11 & -13.03 \\
\hline$(\mathrm{DMA})_{1} \cdot(\mathrm{TFA})_{1}$ & -8.59 & -8.56 & -8.52 & -8.49 & -8.46 \\
\hline$(\mathrm{SA})_{2} \cdot(\mathrm{DMA})_{1}$ & -36.15 & -36.07 & -35.99 & -35.91 & -35.84 \\
\hline$(\mathrm{SA})_{2} \cdot(\mathrm{DMA})_{1} \cdot(\mathrm{TFA})_{1}$ & -41.90 & -41.78 & -41.66 & -41.55 & -41.43 \\
\hline$(\mathrm{SA})_{2} \cdot(\mathrm{DMA})_{2} \cdot(\mathrm{TFA})_{1}$ & -61.57 & -61.41 & -61.24 & -61.07 & -60.91 \\
\hline$(\mathrm{SA})_{2} \cdot(\mathrm{DMA})_{3} \cdot(\mathrm{TFA})_{1}$ & -79.10 & -78.90 & -78.69 & -78.48 & -78.28 \\
\hline$(\mathrm{SA})_{2} \cdot(\mathrm{TFA})_{1}$ & -14.52 & -14.44 & -14.36 & -14.28 & -14.20 \\
\hline$(\mathrm{SA})_{2} \cdot(\mathrm{DMA})_{2}$ & -56.72 & -56.61 & -56.49 & -56.37 & -56.26 \\
\hline$(\mathrm{SA})_{2}$ & -8.98 & -8.94 & -8.91 & -8.87 & -8.84 \\
\hline$(\mathrm{DMA})_{1} \cdot(\mathrm{TFA})_{2}$ & -18.91 & -18.84 & -18.76 & -18.69 & -18.61 \\
\hline$(\mathrm{DMA})_{2} \cdot(\mathrm{TFA})_{2}$ & -34.32 & -34.21 & -34.09 & -33.97 & -33.86 \\
\hline$(\mathrm{TFA})_{2}$ & -5.69 & -5.65 & -5.61 & -5.57 & -5.54 \\
\hline$(\mathrm{SA})_{3} \cdot(\mathrm{DMA})_{1}$ & -49.82 & -49.70 & -49.58 & -49.46 & -49.34 \\
\hline$(\mathrm{SA})_{3} \cdot(\mathrm{DMA})_{2}$ & -73.47 & -73.31 & -73.15 & -73.00 & -72.84 \\
\hline$(\mathrm{SA})_{3} \cdot(\mathrm{DMA})_{3}$ & -93.84 & -93.65 & -93.45 & -93.25 & -93.06 \\
\hline$(\mathrm{SA})_{3}$ & -15.17 & -15.09 & -15.02 & -14.94 & -14.86 \\
\hline$(\mathrm{DMA})_{1} \cdot(\mathrm{TFA})_{3}$ & -22.12 & -21.99 & -21.86 & -21.72 & -21.59 \\
\hline$(\mathrm{DMA})_{2} \cdot(\mathrm{TFA})_{3}$ & -42.80 & -42.64 & -42.47 & -42.31 & -42.14 \\
\hline$(\mathrm{DMA})_{3} \cdot(\mathrm{TFA})_{3}$ & -53.76 & -53.56 & -53.35 & -53.15 & -52.94 \\
\hline$(\mathrm{TFA})_{3}$ & -3.64 & -3.57 & -3.50 & -3.43 & -3.35 \\
\hline
\end{tabular}


Table S10. Continued: Gibbs free formation energies $(\Delta G, \mathrm{kcal} / \mathrm{mol})$ of clusters at different temperatures in different months of the studied cities shown in Table S9.

\begin{tabular}{|c|c|c|c|c|c|}
\hline \multirow{2}{*}{ Clusters } & \multicolumn{5}{|c|}{$\Delta G(\mathrm{kcal} / \mathrm{mol})$} \\
\hline & $287 \mathrm{~K}$ & $288 \mathrm{~K}$ & $289 \mathrm{~K}$ & $290 \mathrm{~K}$ & $291 \mathrm{~K}$ \\
\hline$(\mathrm{SA})_{1} \cdot(\mathrm{DMA})_{1}$ & -15.04 & -15.01 & -14.97 & -14.94 & -14.90 \\
\hline$(\mathrm{SA})_{1} \cdot(\mathrm{DMA})_{1} \cdot(\mathrm{TFA})_{1}$ & -26.70 & -26.63 & -26.56 & -26.48 & -26.41 \\
\hline$(\mathrm{SA})_{1} \cdot(\mathrm{DMA})_{2} \cdot(\mathrm{TFA})_{1}$ & -44.10 & -43.98 & -43.86 & -43.75 & -43.63 \\
\hline$(\mathrm{SA})_{1} \cdot(\mathrm{TFA})_{1}$ & -7.58 & -7.55 & -7.51 & -7.48 & -7.44 \\
\hline$(\mathrm{SA})_{1} \cdot(\mathrm{DMA})_{1} \cdot(\mathrm{TFA})_{2}$ & -35.53 & -35.42 & -35.31 & -35.19 & -35.08 \\
\hline$(\mathrm{SA})_{1} \cdot(\mathrm{DMA})_{2} \cdot(\mathrm{TFA})_{2}$ & -51.24 & -51.07 & -50.91 & -50.74 & -50.57 \\
\hline$(\mathrm{SA})_{1} \cdot(\mathrm{DMA})_{3} \cdot(\mathrm{TFA})_{2}$ & -66.95 & -66.74 & -66.54 & -66.33 & -66.13 \\
\hline$(\mathrm{SA})_{1} \cdot(\mathrm{TFA})_{2}$ & -12.95 & -12.88 & -12.80 & -12.72 & -12.64 \\
\hline$(\mathrm{DMA})_{1} \cdot(\mathrm{TFA})_{1}$ & -8.42 & -8.39 & -8.36 & -8.33 & -8.29 \\
\hline$(\mathrm{SA})_{2} \cdot(\mathrm{DMA})_{1}$ & -35.76 & -35.68 & -35.60 & -35.53 & -35.45 \\
\hline$(\mathrm{SA})_{2} \cdot(\mathrm{DMA})_{1} \cdot(\mathrm{TFA})_{1}$ & -41.31 & -41.20 & -41.08 & -40.96 & -40.84 \\
\hline$(\mathrm{SA})_{2} \cdot(\mathrm{DMA})_{2} \cdot(\mathrm{TFA})_{1}$ & -60.74 & -60.57 & -60.41 & -60.24 & -60.07 \\
\hline$(\mathrm{SA})_{2} \cdot(\mathrm{DMA})_{3} \cdot(\mathrm{TFA})_{1}$ & -78.07 & -77.87 & -77.66 & -77.46 & -77.25 \\
\hline$(\mathrm{SA})_{2} \cdot(\mathrm{TFA})_{1}$ & -14.13 & -14.05 & -13.97 & -13.89 & -13.81 \\
\hline$(\mathrm{SA})_{2} \cdot(\mathrm{DMA})_{2}$ & -56.14 & -56.03 & -55.91 & -55.80 & -55.68 \\
\hline$(\mathrm{SA})_{2}$ & -8.81 & -8.77 & -8.74 & -8.71 & -8.67 \\
\hline$(\mathrm{DMA})_{1} \cdot(\mathrm{TFA})_{2}$ & -18.54 & -18.46 & -18.39 & -18.31 & -18.24 \\
\hline$(\mathrm{DMA})_{2} \cdot(\mathrm{TFA})_{2}$ & -33.74 & -33.63 & -33.51 & -33.40 & -33.28 \\
\hline$(\mathrm{TFA})_{2}$ & -5.50 & -5.46 & -5.42 & -5.39 & -5.35 \\
\hline$(\mathrm{SA})_{3} \cdot(\mathrm{DMA})_{1}$ & -49.22 & -49.10 & -48.99 & -48.87 & -48.75 \\
\hline$(\mathrm{SA})_{3} \cdot(\mathrm{DMA})_{2}$ & -72.69 & -72.53 & -72.37 & -72.22 & -72.06 \\
\hline$(\mathrm{SA})_{3} \cdot(\mathrm{DMA})_{3}$ & -92.86 & -92.66 & -92.47 & -92.27 & -92.07 \\
\hline$(\mathrm{SA})_{3}$ & -14.79 & -14.71 & -14.63 & -14.56 & -14.48 \\
\hline$(\mathrm{DMA})_{1} \cdot(\mathrm{TFA})_{3}$ & -21.46 & -21.32 & -21.19 & -21.06 & -20.93 \\
\hline$(\mathrm{DMA})_{2} \cdot(\mathrm{TFA})_{3}$ & -41.98 & -41.81 & -41.65 & -41.49 & -41.32 \\
\hline$(\mathrm{DMA})_{3} \cdot(\mathrm{TFA})_{3}$ & -52.73 & -52.53 & -52.32 & -52.12 & -51.91 \\
\hline$(\mathrm{TFA})_{3}$ & -3.28 & -3.21 & -3.14 & -3.06 & -2.99 \\
\hline
\end{tabular}


Table S10. Continued: Gibbs free formation energies $(\Delta G, \mathrm{kcal} / \mathrm{mol})$ of clusters at different temperatures in different months of the studied cities shown in Table S9.

\begin{tabular}{|c|c|c|c|c|c|}
\hline \multirow{2}{*}{ Clusters } & \multicolumn{5}{|c|}{$\Delta G(\mathrm{kcal} / \mathrm{mol})$} \\
\hline & $292 \mathrm{~K}$ & $293 \mathrm{~K}$ & $294 \mathrm{~K}$ & $295 \mathrm{~K}$ & $296 \mathrm{~K}$ \\
\hline$(\mathrm{SA})_{1} \cdot(\mathrm{DMA})_{1}$ & -14.87 & -14.84 & -14.80 & -14.77 & -14.73 \\
\hline$(\mathrm{SA})_{1} \cdot(\mathrm{DMA})_{1} \cdot(\mathrm{TFA})_{1}$ & -26.34 & -26.26 & -26.19 & -26.12 & -26.05 \\
\hline$(\mathrm{SA})_{1} \cdot(\mathrm{DMA})_{2} \cdot(\mathrm{TFA})_{1}$ & -43.51 & -43.40 & -43.28 & -43.16 & -43.05 \\
\hline$(\mathrm{SA})_{1} \cdot(\mathrm{TFA})_{1}$ & -7.41 & -7.37 & -7.34 & -7.30 & -7.27 \\
\hline$(\mathrm{SA})_{1} \cdot(\mathrm{DMA})_{1} \cdot(\mathrm{TFA})_{2}$ & -34.97 & -34.86 & -34.74 & -34.63 & -34.52 \\
\hline$(\mathrm{SA})_{1} \cdot(\mathrm{DMA})_{2} \cdot(\mathrm{TFA})_{2}$ & -50.41 & -50.24 & -50.08 & -49.91 & -49.74 \\
\hline$(\mathrm{SA})_{1} \cdot(\mathrm{DMA})_{3} \cdot(\mathrm{TFA})_{2}$ & -65.92 & -65.72 & -65.51 & -65.31 & -65.10 \\
\hline$(\mathrm{SA})_{1} \cdot(\mathrm{TFA})_{2}$ & -12.57 & -12.49 & -12.41 & -12.34 & -12.26 \\
\hline$(\mathrm{DMA})_{1} \cdot(\mathrm{TFA})_{1}$ & -8.26 & -8.23 & -8.19 & -8.16 & -8.13 \\
\hline$(\mathrm{SA})_{2} \cdot(\mathrm{DMA})_{1}$ & -35.37 & -35.29 & -35.22 & -35.14 & -35.06 \\
\hline$(\mathrm{SA})_{2} \cdot(\mathrm{DMA})_{1} \cdot(\mathrm{TFA})_{1}$ & -40.73 & -40.61 & -40.49 & -40.38 & -40.26 \\
\hline$(\mathrm{SA})_{2} \cdot(\mathrm{DMA})_{2} \cdot(\mathrm{TFA})_{1}$ & -59.91 & -59.74 & -59.57 & -59.41 & -59.24 \\
\hline$(\mathrm{SA})_{2} \cdot(\mathrm{DMA})_{3} \cdot(\mathrm{TFA})_{1}$ & -77.05 & -76.84 & -76.64 & -76.43 & -76.23 \\
\hline$(\mathrm{SA})_{2} \cdot(\mathrm{TFA})_{1}$ & -13.73 & -13.65 & -13.57 & -13.49 & -13.41 \\
\hline$(\mathrm{SA})_{2} \cdot(\mathrm{DMA})_{2}$ & -55.57 & -55.45 & -55.34 & -55.22 & -55.11 \\
\hline$(\mathrm{SA})_{2}$ & -8.64 & -8.60 & -8.57 & -8.54 & -8.50 \\
\hline$(\mathrm{DMA})_{1} \cdot(\mathrm{TFA})_{2}$ & -18.16 & -18.09 & -18.01 & -17.94 & -17.86 \\
\hline$(\mathrm{DMA})_{2} \cdot(\mathrm{TFA})_{2}$ & -33.16 & -33.05 & -32.93 & -32.82 & -32.70 \\
\hline$(\mathrm{TFA})_{2}$ & -5.31 & -5.27 & -5.24 & -5.20 & -5.16 \\
\hline$(\mathrm{SA})_{3} \cdot(\mathrm{DMA})_{1}$ & -48.63 & -48.51 & -48.39 & -48.27 & -48.16 \\
\hline$(\mathrm{SA})_{3} \cdot(\mathrm{DMA})_{2}$ & -71.91 & -71.75 & -71.60 & -71.44 & -71.29 \\
\hline$(\mathrm{SA})_{3} \cdot(\mathrm{DMA})_{3}$ & -91.88 & -91.68 & -91.49 & -91.29 & -91.09 \\
\hline$(\mathrm{SA})_{3}$ & -14.40 & -14.32 & -14.25 & -14.17 & -14.10 \\
\hline$(\mathrm{DMA})_{1} \cdot(\mathrm{TFA})_{3}$ & -20.80 & -20.66 & -20.53 & -20.40 & -20.26 \\
\hline$(\mathrm{DMA})_{2} \cdot(\mathrm{TFA})_{3}$ & -41.16 & -41.00 & -40.83 & -40.67 & -40.51 \\
\hline$(\mathrm{DMA})_{3} \cdot(\mathrm{TFA})_{3}$ & -51.71 & -51.50 & -51.29 & -51.09 & -50.88 \\
\hline$(\mathrm{TFA})_{3}$ & -2.92 & -2.85 & -2.78 & -2.70 & -2.63 \\
\hline
\end{tabular}


Table S10. Continued: Gibbs free formation energies $(\Delta G, \mathrm{kcal} / \mathrm{mol})$ of clusters at different temperatures in different months of the studied cities shown in Table S9.

\begin{tabular}{|c|c|c|c|c|c|}
\hline \multirow{2}{*}{ Clusters } & \multicolumn{5}{|c|}{$\Delta G(\mathrm{kcal} / \mathrm{mol})$} \\
\hline & $297 \mathrm{~K}$ & $298 \mathrm{~K}$ & $299 \mathrm{~K}$ & $301 \mathrm{~K}$ & $303 \mathrm{~K}$ \\
\hline$(\mathrm{SA})_{1} \cdot(\mathrm{DMA})_{1}$ & -14.70 & -14.66 & -14.63 & -14.56 & -14.49 \\
\hline$(\mathrm{SA})_{1} \cdot(\mathrm{DMA})_{1} \cdot(\mathrm{TFA})_{1}$ & -25.97 & -25.90 & -25.83 & -25.68 & -25.54 \\
\hline$(\mathrm{SA})_{1} \cdot(\mathrm{DMA})_{2} \cdot(\mathrm{TFA})_{1}$ & -42.93 & -42.81 & -42.69 & -42.46 & -42.23 \\
\hline$(\mathrm{SA})_{1} \cdot(\mathrm{TFA})_{1}$ & -7.23 & -7.20 & -7.16 & -7.09 & -7.02 \\
\hline$(\mathrm{SA})_{1} \cdot(\mathrm{DMA})_{1} \cdot(\mathrm{TFA})_{2}$ & -34.41 & -34.29 & -34.18 & -33.96 & -33.73 \\
\hline$(\mathrm{SA})_{1} \cdot(\mathrm{DMA})_{2} \cdot(\mathrm{TFA})_{2}$ & -49.58 & -49.41 & -49.25 & -48.91 & -48.58 \\
\hline$(\mathrm{SA})_{1} \cdot(\mathrm{DMA})_{3} \cdot(\mathrm{TFA})_{2}$ & -64.90 & -64.69 & -64.49 & -64.08 & -63.67 \\
\hline$(\mathrm{SA})_{1} \cdot(\mathrm{TFA})_{2}$ & -12.18 & -12.11 & -12.03 & -11.88 & -11.72 \\
\hline$(\mathrm{DMA})_{1} \cdot(\mathrm{TFA})_{1}$ & -8.10 & -8.06 & -8.03 & -7.96 & -7.90 \\
\hline$(\mathrm{SA})_{2} \cdot(\mathrm{DMA})_{1}$ & -34.98 & -34.91 & -34.83 & -34.68 & -34.52 \\
\hline$(\mathrm{SA})_{2} \cdot(\mathrm{DMA})_{1} \cdot(\mathrm{TFA})_{1}$ & -40.14 & -40.03 & -39.91 & -39.68 & -39.44 \\
\hline$(\mathrm{SA})_{2} \cdot(\mathrm{DMA})_{2} \cdot(\mathrm{TFA})_{1}$ & -59.07 & -58.91 & -58.74 & -58.41 & -58.07 \\
\hline$(\mathrm{SA})_{2} \cdot(\mathrm{DMA})_{3} \cdot(\mathrm{TFA})_{1}$ & -76.02 & -75.81 & -75.61 & -75.20 & -74.79 \\
\hline$(\mathrm{SA})_{2} \cdot(\mathrm{TFA})_{1}$ & -13.33 & -13.25 & -13.18 & -13.02 & -12.86 \\
\hline$(\mathrm{SA})_{2} \cdot(\mathrm{DMA})_{2}$ & -54.99 & -54.88 & -54.76 & -54.53 & -54.30 \\
\hline$(\mathrm{SA})_{2}$ & -8.47 & -8.44 & -8.40 & -8.34 & -8.27 \\
\hline$(\mathrm{DMA})_{1} \cdot(\mathrm{TFA})_{2}$ & -17.79 & -17.71 & -17.64 & -17.49 & -17.34 \\
\hline$(\mathrm{DMA})_{2} \cdot(\mathrm{TFA})_{2}$ & -32.59 & -32.47 & -32.36 & -32.13 & -31.89 \\
\hline$(\mathrm{TFA})_{2}$ & -5.13 & -5.09 & -5.05 & -4.97 & -4.90 \\
\hline$(\mathrm{SA})_{3} \cdot(\mathrm{DMA})_{1}$ & -48.04 & -47.92 & -47.80 & -47.56 & -47.32 \\
\hline$(\mathrm{SA})_{3} \cdot(\mathrm{DMA})_{2}$ & -71.13 & -70.97 & -70.82 & -70.51 & -70.19 \\
\hline$(\mathrm{SA})_{3} \cdot(\mathrm{DMA})_{3}$ & -90.90 & -90.70 & -90.50 & -90.11 & -89.72 \\
\hline$(\mathrm{SA})_{3}$ & -14.02 & -13.94 & -13.86 & -13.71 & -13.56 \\
\hline$(\mathrm{DMA})_{1} \cdot(\mathrm{TFA})_{3}$ & -20.13 & -20.00 & -19.87 & -19.60 & -19.34 \\
\hline$(\mathrm{DMA})_{2} \cdot(\mathrm{TFA})_{3}$ & -40.34 & -40.18 & -40.01 & -39.69 & -39.36 \\
\hline$(\mathrm{DMA})_{3} \cdot(\mathrm{TFA})_{3}$ & -50.68 & -50.47 & -50.27 & -49.86 & -49.44 \\
\hline$(\mathrm{TFA})_{3}$ & -2.56 & -2.49 & -2.41 & -2.27 & -2.13 \\
\hline
\end{tabular}


Table S10. Continued: Gibbs free formation energies $(\Delta G, \mathrm{kcal} / \mathrm{mol})$ of clusters at different temperatures in different months of the studied cities shown in Table S9.

\begin{tabular}{|c|c|c|c|c|c|}
\hline \multirow{2}{*}{ Clusters } & \multicolumn{5}{|c|}{$\Delta G(\mathrm{kcal} / \mathrm{mol})$} \\
\hline & $304 \mathrm{~K}$ & $306 \mathrm{~K}$ & $307 \mathrm{~K}$ & $308 \mathrm{~K}$ & $309 \mathrm{~K}$ \\
\hline$(\mathrm{SA})_{1} \cdot(\mathrm{DMA})_{1}$ & -14.46 & -14.39 & -14.35 & -14.32 & -14.29 \\
\hline$(\mathrm{SA})_{1} \cdot(\mathrm{DMA})_{1} \cdot(\mathrm{TFA})_{1}$ & -25.47 & -25.32 & -25.25 & -25.18 & -25.10 \\
\hline$(\mathrm{SA})_{1} \cdot(\mathrm{DMA})_{2} \cdot(\mathrm{TFA})_{1}$ & -42.11 & -41.88 & -41.76 & -41.64 & -41.53 \\
\hline$(\mathrm{SA})_{1} \cdot(\mathrm{TFA})_{1}$ & -6.99 & -6.92 & -6.88 & -6.85 & -6.81 \\
\hline$(\mathrm{SA})_{1} \cdot(\mathrm{DMA})_{1} \cdot(\mathrm{TFA})_{2}$ & -33.62 & -33.40 & -33.28 & -33.17 & -33.06 \\
\hline$(\mathrm{SA})_{1} \cdot(\mathrm{DMA})_{2} \cdot(\mathrm{TFA})_{2}$ & -48.42 & -48.08 & -47.92 & -47.75 & -47.59 \\
\hline$(\mathrm{SA})_{1} \cdot(\mathrm{DMA})_{3} \cdot(\mathrm{TFA})_{2}$ & -63.46 & -63.05 & -62.85 & -62.64 & -62.44 \\
\hline$(\mathrm{SA})_{1} \cdot(\mathrm{TFA})_{2}$ & -11.65 & -11.49 & -11.42 & -11.34 & -11.26 \\
\hline$(\mathrm{DMA})_{1} \cdot(\mathrm{TFA})_{1}$ & -7.87 & -7.80 & -7.77 & -7.74 & -7.70 \\
\hline$(\mathrm{SA})_{2} \cdot(\mathrm{DMA})_{1}$ & -34.44 & -34.29 & -34.21 & -34.13 & -34.06 \\
\hline$(\mathrm{SA})_{2} \cdot(\mathrm{DMA})_{1} \cdot(\mathrm{TFA})_{1}$ & -39.33 & -39.09 & -38.98 & -38.86 & -38.74 \\
\hline$(\mathrm{SA})_{2} \cdot(\mathrm{DMA})_{2} \cdot(\mathrm{TFA})_{1}$ & -57.91 & -57.58 & -57.41 & -57.24 & -57.08 \\
\hline$(\mathrm{SA})_{2} \cdot(\mathrm{DMA})_{3} \cdot(\mathrm{TFA})_{1}$ & -74.58 & -74.17 & -73.97 & -73.76 & -73.56 \\
\hline$(\mathrm{SA})_{2} \cdot(\mathrm{TFA})_{1}$ & -12.78 & -12.62 & -12.54 & -12.46 & -12.39 \\
\hline$(\mathrm{SA})_{2} \cdot(\mathrm{DMA})_{2}$ & -54.19 & -53.96 & -53.84 & -53.73 & -53.61 \\
\hline$(\mathrm{SA})_{2}$ & -8.24 & -8.17 & -8.13 & -8.10 & -8.07 \\
\hline$(\mathrm{DMA})_{1} \cdot(\mathrm{TFA})_{2}$ & -17.27 & -17.12 & -17.04 & -16.97 & -16.89 \\
\hline$(\mathrm{DMA})_{2} \cdot(\mathrm{TFA})_{2}$ & -31.78 & -31.55 & -31.43 & -31.32 & -31.20 \\
\hline$(\mathrm{TFA})_{2}$ & -4.86 & -4.79 & -4.75 & -4.71 & -4.68 \\
\hline$(\mathrm{SA})_{3} \cdot(\mathrm{DMA})_{1}$ & -47.21 & -46.97 & -46.85 & -46.73 & -46.61 \\
\hline$(\mathrm{SA})_{3} \cdot(\mathrm{DMA})_{2}$ & -70.04 & -69.73 & -69.57 & -69.42 & -69.26 \\
\hline$(\mathrm{SA})_{3} \cdot(\mathrm{DMA})_{3}$ & -89.52 & -89.13 & -88.94 & -88.74 & -88.54 \\
\hline$(\mathrm{SA})_{3}$ & -13.48 & -13.33 & -13.25 & -13.17 & -13.10 \\
\hline$(\mathrm{DMA})_{1} \cdot(\mathrm{TFA})_{3}$ & -19.21 & -18.94 & -18.81 & -18.68 & -18.55 \\
\hline$(\mathrm{DMA})_{2} \cdot(\mathrm{TFA})_{3}$ & -39.20 & -38.87 & -38.71 & -38.54 & -38.38 \\
\hline$(\mathrm{DMA})_{3} \cdot(\mathrm{TFA})_{3}$ & -49.24 & -48.83 & -48.62 & -48.42 & -48.21 \\
\hline$(\mathrm{TFA})_{3}$ & -2.05 & -1.91 & -1.84 & -1.76 & -1.69 \\
\hline
\end{tabular}



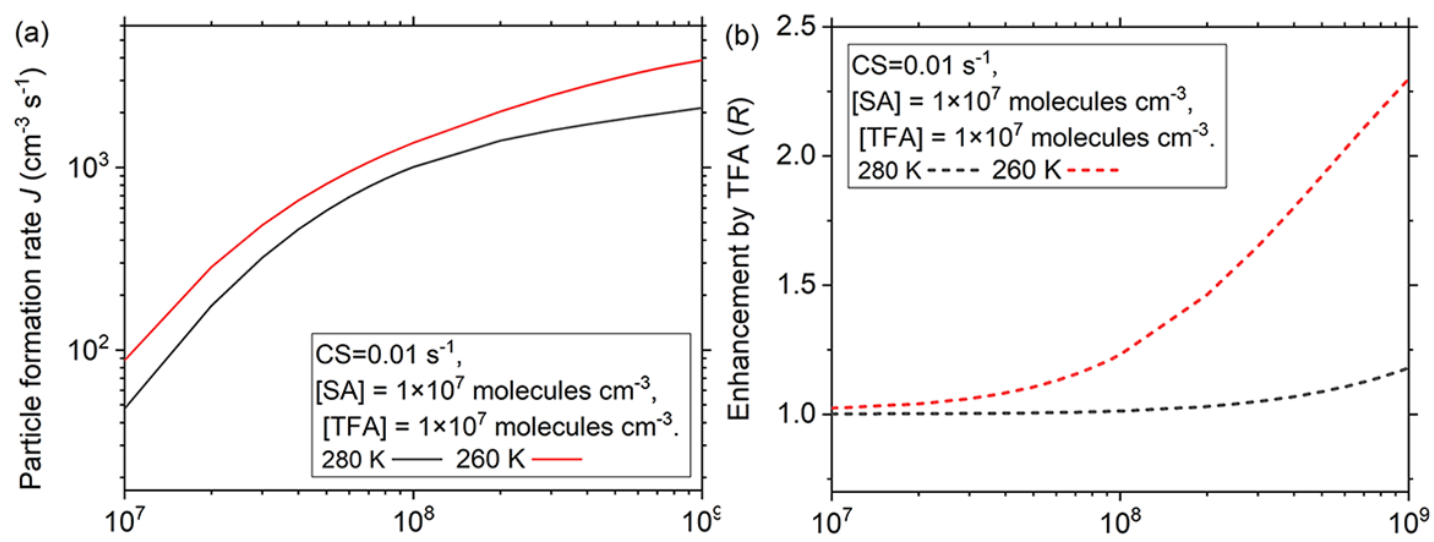

DMA monomer concentration (molecules $\mathrm{cm}^{-3}$ )
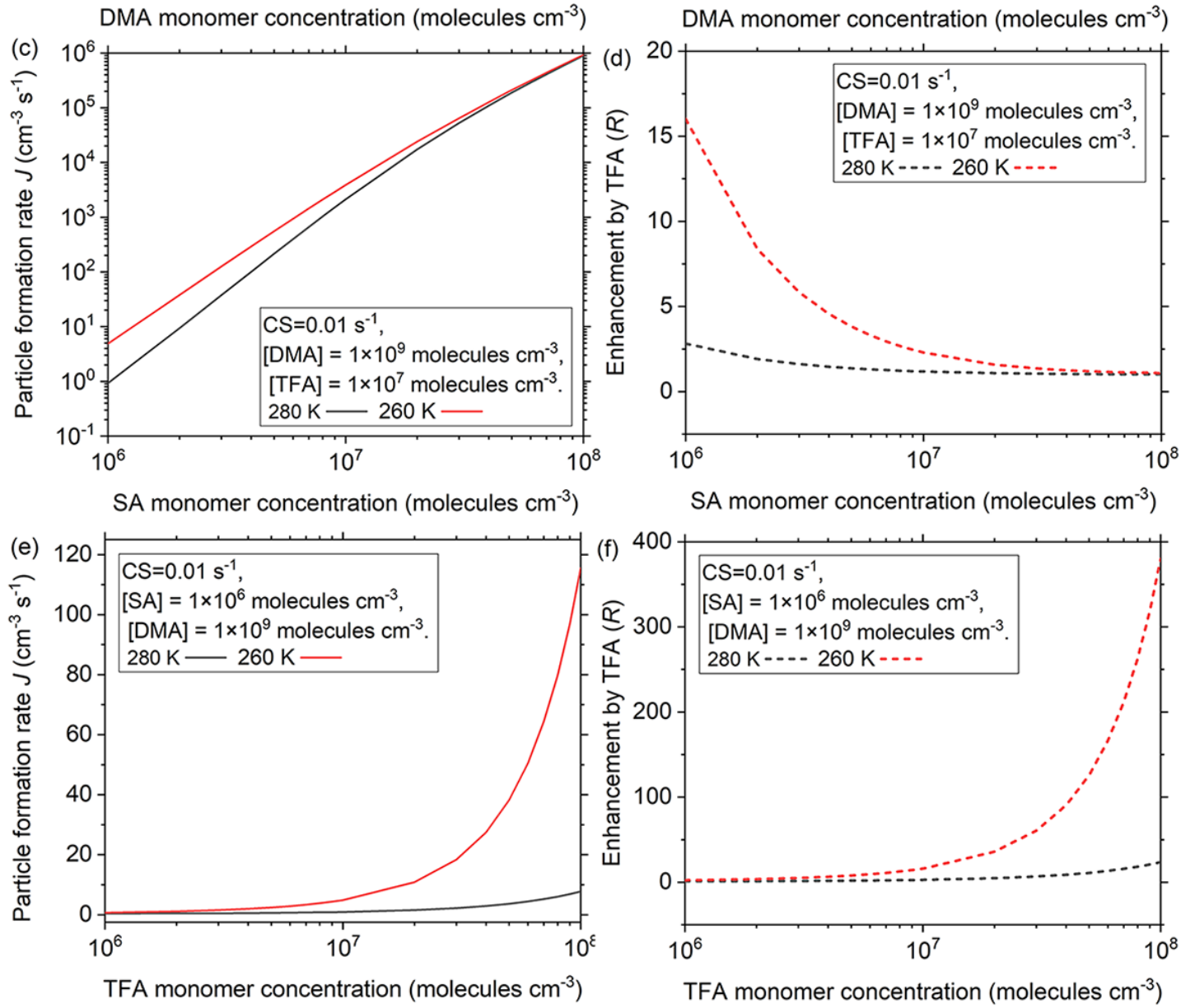

$\mathrm{SA}$ monomer concentration (molecules $\mathrm{cm}^{-3}$ )

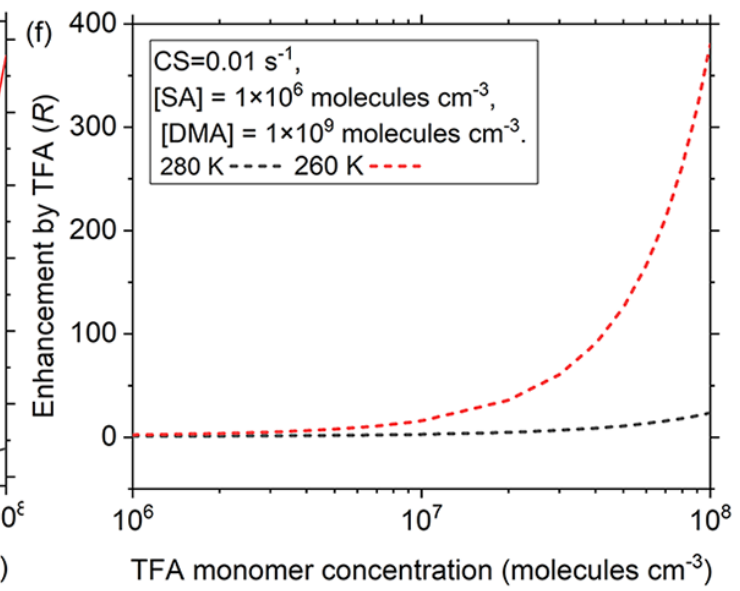

Figure S1. Particle formation rates $\left(J, \mathrm{~cm}^{-3} \mathrm{~s}^{-1}\right)$ at different temperatures $(280 \mathrm{~K}$ and $260 \mathrm{~K})$ as a function of (a) DMA monomer concentrations, (c) SA monomer concentrations and (e) TFA monomer concentrations. Enhancement of particle formation rate by TFA $\left(R, R=J_{\mathrm{SA}-\mathrm{DMA}-\mathrm{TFA}} / J_{\mathrm{SA}-}\right.$ DMA) at different temperatures as a function of (b) DMA monomer concentrations, (d) SA monomer concentrations and (f) TFA monomer concentrations. Black and red lines are corresponding to 280 $\mathrm{K}$ and $260 \mathrm{~K}$, respectively. $\mathrm{CS}=0.01 \mathrm{~s}^{-1}$. 

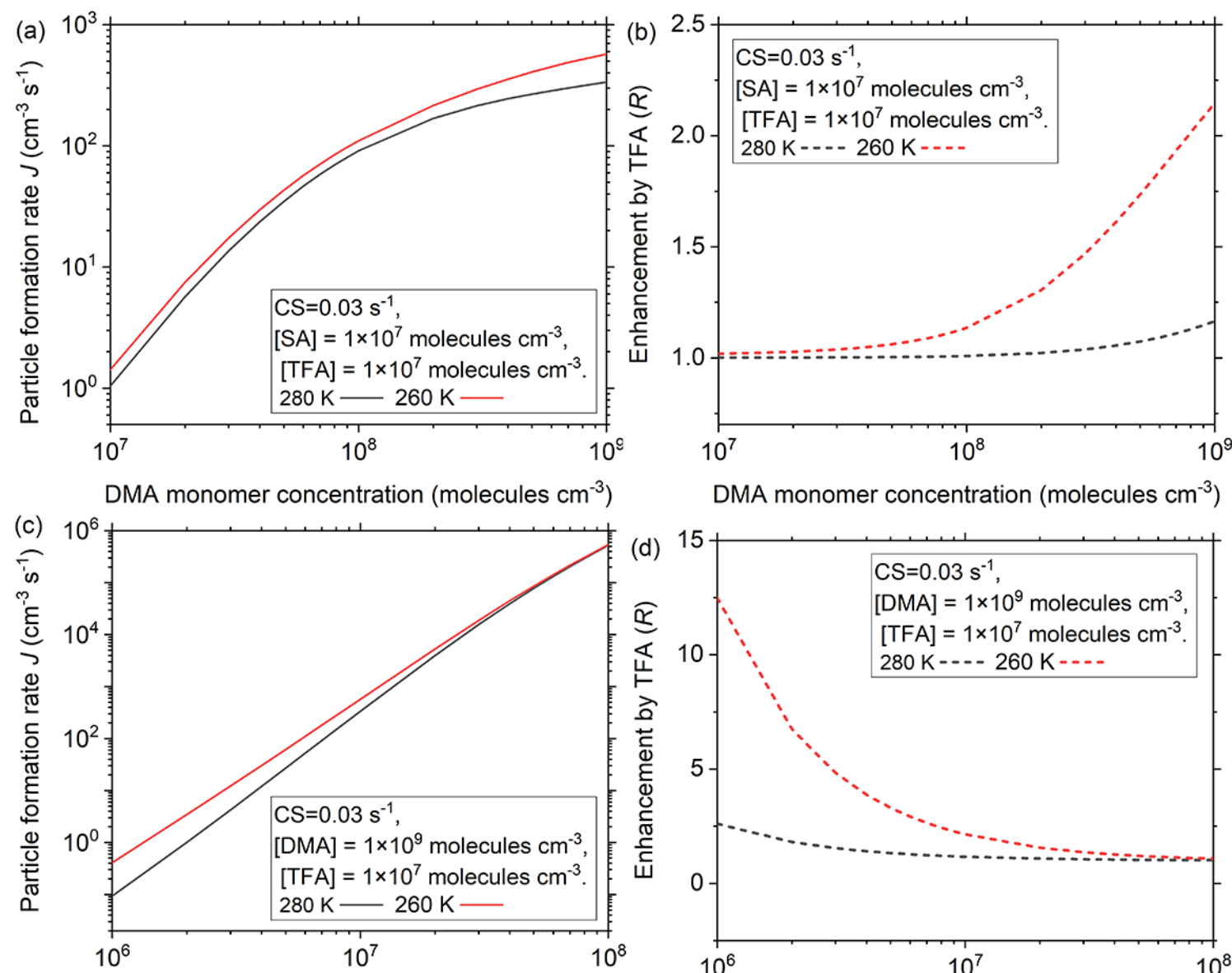

DMA monomer concentration (molecules $\mathrm{cm}^{-3}$ )

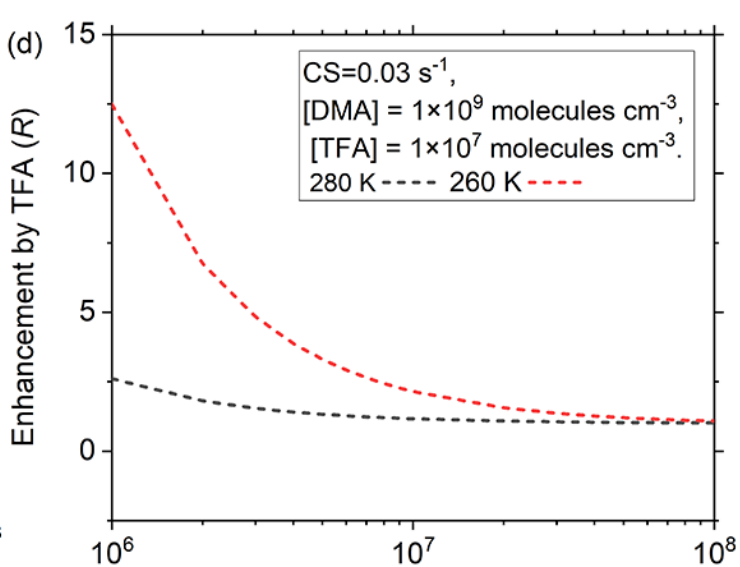

$\mathrm{SA}$ monomer concentration (molecules $\mathrm{cm}^{-3}$ )

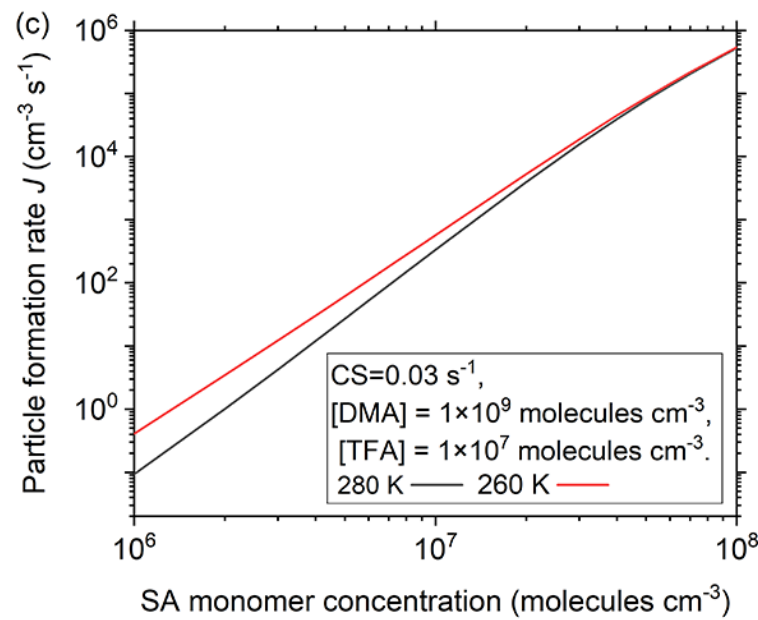

SA monomer concentration (molecules $\mathrm{cm}^{-3}$ )

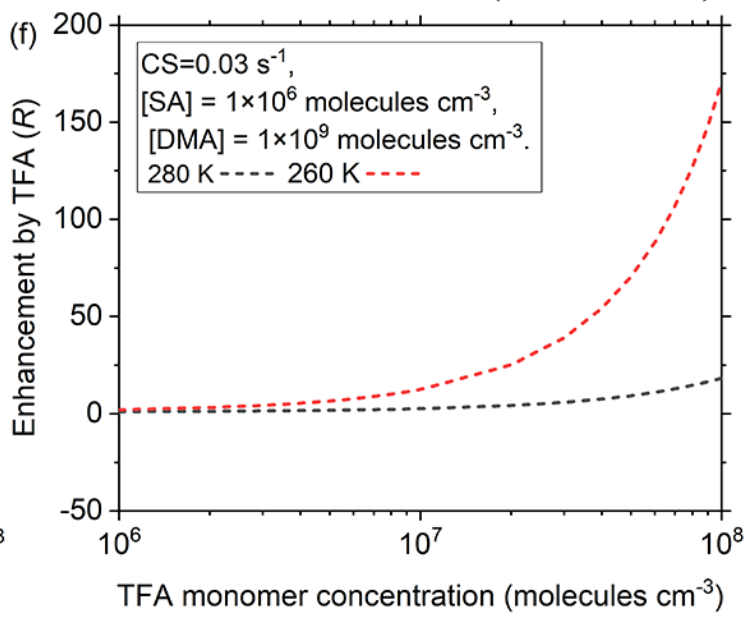

Figure S2. Particle formation rates $\left(J, \mathrm{~cm}^{-3} \mathrm{~s}^{-1}\right)$ at different temperatures $(280 \mathrm{~K}$ and $260 \mathrm{~K})$ as a function of (a) DMA monomer concentrations, (c) SA monomer concentrations and (e) TFA monomer concentrations. Enhancement of particle formation rate by TFA $\left(R, R=J_{\mathrm{SA}-\mathrm{DMA}-\mathrm{TFA}} / J_{\mathrm{SA}-}\right.$ DMA) at different temperatures as a function of (b) DMA monomer concentrations, (d) SA monomer concentrations and (f) TFA monomer concentrations. Black and red lines are corresponding to 280 $\mathrm{K}$ and $260 \mathrm{~K}$, respectively. $\mathrm{CS}=0.03 \mathrm{~s}^{-1}$. 


\section{References}

Elm, J.: Elucidating the limiting steps in sulfuric acid-base new particle formation, J. Phys. Chem. A, 121(43), 8288-8295, doi:10.1021/acs.jpca.7b08962, 2017.

Lu, Y., Liu, L., Ning, A., Yang, G., Liu, Y., Kurtén, T., Vehkamäki, H., Zhang, X. and Wang, L.: Atmospheric sulfuric acid-dimethylamine nucleation enhanced by trifluoroacetic acid, Geophys. Res. Lett., 47(2), doi:10.1029/2019g1085627, 2020.

Olenius, T., Halonen, R., Kurtén, T., Henschel, H., Kupiainen-Määttä, O., Ortega, I. K., Jen, C. N., Vehkamäki, H. and Riipinen, I.: New particle formation from sulfuric acid and amines: Comparison of monomethylamine, dimethylamine, and trimethylamine, J. Geophys. Res., 122(13), 7103-7118, doi:10.1002/2017JD026501, 2017.

Paasonen, P., Olenius, T., Kupiainen, O., Kurtén, T., Petäjä, T., Birmili, W., Hamed, A., Hu, M., Huey, L. G., Plass-Duelmer, C., Smith, J. N., Wiedensohler, A., Loukonen, V., McGrath, M. J., Ortega, I. K., Laaksonen, A., Vehkamäki, H., Kerminen, V.-M. and Kulmala, M.: On the formation of sulphuric acid-amine clusters in varying atmospheric conditions and its influence on atmospheric new particle formation, Atmos. Chem. Phys., 12(19), 9113-9133, doi:10.5194/acp12-9113-2012, 2012. 